\title{
The ROSAT Galactic Plane Survey: Analysis of a low latitude sample area in Cygnus ${ }^{\star}$
}

\section{The observations}

\author{
C. Motch ${ }^{1,2}$, P. Guillout ${ }^{1}$, F. Haberl ${ }^{2}$, M.W. Pakull ${ }^{1}$, W. Pietsch ${ }^{2}$, and K. Reinsch ${ }^{3}$ \\ 1 Observatoire Astronomique, UA 1280 CNRS, 11 rue de l'Université, F-67000 Strasbourg, France \\ 2 Max-Planck-Institut für Extraterrestrische Physik, D-85740, Garching bei München, Germany \\ 3 Sternwarte, Geismarlandstrasse 11, D-37083, Göttingen, Germany
}

Received March 20; accepted April 22, 1996

\begin{abstract}
The analysis of the part of the ROSAT all-sky survey covering the galactic plane is the scope of a dedicated project called the ROSAT Galactic Plane Survey. In order to statistically understand the nature of the $\approx 14000$ sources discovered by ROSAT at $|b| \leq 20^{\circ}$, a number of sample areas have been chosen for follow-up optical identification. In this paper we present the X-ray and optical material gathered in a region located in the Cygnus constellation, centered at $l=90^{\circ}, b=0^{\circ}$ and covering an area of $64.5 \mathrm{deg}^{2}$. A total of 95 and 128 sources are detected with a maximum likelihood larger than 10 and 8 respectively. With a typical survey exposure time of the order of 700 to $900 \mathrm{~s}$ the flux completeness level is $\approx 0.02 \mathrm{cts} \mathrm{s}^{-1}$ corresponding to $\approx 210^{-13} \mathrm{erg} \mathrm{cm}^{-2} \mathrm{~s}^{-1}$. The position of the sample area allows to investigate the soft X-ray content of a rather typical region of the galactic plane. In this paper we describe the details of the observational procedures and data reduction. For each ROSAT source we list the main X-ray characteristics together with those of the proposed optical identification. When appropriate, we also show optical spectra and finding charts. The full analysis and discussion of these data are presented in a companion paper (Motch et al. 1997). ${ }^{1}$
\end{abstract}

Key words: X-ray general, X-ray stars, stars: activity, stars: neutron, stars: statistics

Send offprint requests to: C. Motch

* Partly based on observations obtained at the Observatoire de Haute-Provence, CNRS, France.

1 Tables 2 to 6 are also available in electronic form at the CDS via anonymous ftp cdsarc.u-strasbg.fr. Figures are only published electronically and are made available at http://www.edphys.fr/Abstract.html

\section{Introduction}

The ROSAT all-sky survey (RASS; Voges 1992) was carried out during the first part of the ROSAT mission from 1990 July till 1991 February. Two instrumentations, the X-ray Telescope (XRT; $0.07-2.4 \mathrm{keV}$ ) and the Wide Field Camera were used at this occasion (Trümper 1983). The ROSAT XRT survey is the first X-ray survey ever made with an imaging instrument and thus offers an improved median sensitivity $\left(2-410^{-13} \mathrm{erg} \mathrm{cm}^{-2} \mathrm{~s}^{-1}\right)$ compared to previous non imaging devices (e.g., HEAO; Nugent et al. 1983; Wood et al. 1984, EXOSAT; Warwick et al. 1985). The analysis of the part of the all-sky survey restricted to absolute galactic latitude below $20^{\circ}$ is the scope of a dedicated project, the ROSAT Galactic Plane Survey (RGPS: Motch et al. 1991). More than 14000 sources, most of them new, are detected in the RGPS area by the Standard Analysis Software System (SASS; Voges et al. 1992). About $20 \%$ to $30 \%$ of the RGPS sources may be identified with high confidence on the basis of positional coincidence with objects, mostly stars, catalogued in the SIMBAD database. Owing to the large number of sources left, two paths of investigations were chosen; i) selection of sources over the whole galactic sky using criteria on their $\mathrm{X}$-ray characteristics and optical content of the error box and ii) selection of sample areas at judicious positions for as complete as possible optical identification.

We present in this paper the X-ray and optical observational material concerning $158 \mathrm{X}$-ray detections in a $64.5 \mathrm{deg}^{2}$ sample area centered at $l=90^{\circ}$ and $b=0^{\circ}$. We also describe the various optical instrumentations used for this project. Located in the Cygnus constellation, the region is void of nearby open clusters and is representative of the typical mid longitude galactic plane. For each source we list in Tables 2 and 3, X-ray position, count rate, hardness ratio and in Tables 4 and 5 the class of the proposed optical counterpart, name, spectral type, magnitudes, 
optical position and distance to X-ray position. When applicable, finding charts and optical spectra of candidate objects are also printed. The analysis and discussion of these data are presented in a companion paper (Motch et al. 1997).

\section{X-ray survey observations}

The detector used for the XRT survey was the Position Sensitive Proportional Counter (PSPC) which offers a spatial resolution of $\approx 20^{\prime \prime}$ and medium energy resolution ( $40 \%$ at $1 \mathrm{keV}$ ) in the range of 0.07 to $2.4 \mathrm{keV}$ (Pfeffermann et al. 1986). During survey observations, the sky was scanned in great circles bound to be perpendicular to the direction to the Sun thus covering the whole celestial sphere in six months. A given part of the sky was seen several times at $96 \mathrm{~min}$ intervals corresponding to the ROSAT orbit and scan period. For a given position on the sky, the number of available scans and total exposure time depends on the ecliptic latitude, the poles being the most deeply observed, and on possible detector switch off episodes (caused by radiation belts) close to the earth magnetic poles and South Atlantic Anomaly. With the field of view of $2^{\circ}$ of the PSPC, each source was seen during a maximum of $32 \mathrm{~s}$ each orbit. Because of the blur at large off axis angles, the average survey point spread function $\left(\mathrm{FWHM} \approx 3^{\prime}\right)$ is significantly larger than the one on-axis but still allows source positioning with a $1 \sigma$ accuracy better than 20" (Voges 1992).

The area chosen for our investigations was a rectangle extending from $l=86^{\circ}$ to $94^{\circ}$ and from $b=-5^{\circ}$ to $+5^{\circ}$. However, part of the selected X-ray region is contaminated by hard diffuse emission from the Cygnus super bubble (Cash et al. 1980) and after rejection of the high background areas the final size of the region was $64.5 \mathrm{deg}^{2}$. Actual area boundaries are described in detail in Motch et al. (1997). By chance, the selected region is located in a range of galactic longitude which is closest to the north ecliptic pole. A strong gradient in exposure time is present in the selected field from about $500 \mathrm{~s}$ at $b=-5^{\circ}$ to $1000 \mathrm{~s}$ at $b=5^{\circ}$. However, $84 \%$ of the sources in our field have exposure times ranging from 700 to $900 \mathrm{~s}$.

The final list of sources was extracted from the merged survey data using the dedicated Extended Scientific Analysis System (EXSAS) developed at MPE (Zimmermann et al. 1992). Source detection was run separately in three energy bands (soft, $0.1-0.4 \mathrm{keV}$; hard, $0.5-2.0 \mathrm{keV}$ and broad, $0.1-2.0 \mathrm{keV}$ ) using the maximum likelihood $(M L)$ algorithm and accepting sources above $M L=7$. The three source lists were then merged into a single one in which we defined the "merged" source count rate and "merged" maximum likelihood as being the maximum of the count rates and maximum likelihood respectively in all three bands. The retained X-ray coordinates were those of the broad band detection or those of the hard or soft bands when the source was not de- tected in the broad band. A total of 95 and 128 sources were detected above $M L=10$ and 8 respectively and 30 additional sources had $M L$ in the range of 7 to 8 . Each of the sources with $M L \geq 8$ has an associated index running from 1 to 128 , increasing with decreasing count rates. Because of the expected high fraction of spurious sources with $7 \leq M L<8$ we do not discuss these marginal detections in detail. We list separately these doubtful detections which were given index numbers in the range of 129 to 158 .

The $90 \%$ confidence error radius was estimated assuming that the uncertainty on the source localization could be expressed as the quadratic sum of the statistical error determined by the maximum likelihood detection algorithm and of a systematic bore sight error of $8^{\prime \prime}$. For a subset of sources, standard ROSAT hardness ratios were computed. We defined hardness ratios 1 and 2 with energy boundaries as used in the latest SASS versions:

$$
\begin{aligned}
& \mathrm{HR} 1=\frac{(0.5-2.0)-(0.1-0.4)}{(0.1-0.4)+(0.5-2.0)} \\
& \mathrm{HR} 2=\frac{(1.0-2.0)-(0.5-1.0)}{(1.0-2.0)}
\end{aligned}
$$

where $(A-B)$ is the raw background corrected source count rate in the $A-B$ energy range expressed in $\mathrm{keV}$. Tables 2 and 3 summarize the main X-ray properties of each detected ROSAT source. From the bending of the $\log N(>S)-\log S$ curve we estimate that at $M L=10$ our completeness level is $0.02 \mathrm{cts} \mathrm{s}^{-1}$ which roughly corresponds to a flux limit of $210^{-13} \mathrm{erg} \mathrm{cm}^{-2}$ $\mathrm{s}^{-1}$. At this flux level the source density is $\approx 1.0$ source $\operatorname{deg}^{-2}$. Using $M L=8$ may allow to improve on the sensitivity (see Motch et al. 1997) and at an estimated completeness level of $0.012 \mathrm{cts} \mathrm{s}^{-1}$ the source density is $\approx 1.8 \mathrm{deg}^{-2}$. However, the enhanced number of spurious sources complicates the definition of a completeness level at $M L=8$.

\section{Optical observations}

All the optical material was collected at the Observatoire de Haute-Provence, CNRS, France. Observations took place during several runs spanning from 1991 May till 1993 September. This observing programme was part of a general project aiming at the optical identification and follow-up observations of area and X-ray selected sources extracted from the RGPS. All spectral and photometric data reductions were performed using standard MIDAS procedures (Banse et al. 1983).

Spectroscopic observations were obtained with the CARELEC spectrograph (Lemaitre et al. 1990) attached at the $1.9 \mathrm{~m}$ telescope. Several CCDs have been used during the course of the observations (see Table 1). Low resolution spectroscopy $(\lambda \lambda 3500-7500 \AA$; FWHM resolution $\approx 14 \AA$ ) and medium resolution blue spectroscopy $(\lambda \lambda 3800-4300 \AA ;$ FWHM resolution $\approx 1.8 \AA)$ 
were acquired using a $260 \AA / \mathrm{mm}$ grating and $33 \AA / \mathrm{mm}$ grating, respectively. Two-dimensional spectra were corrected for bias and flat-field. Extracted spectra were calibrated in wavelength using arcs of iron and helium lamps. Observations of standard stars allowed to formally calibrate in flux all spectra. However, bad meteorological conditions and the narrow slit entrance width used for some bright stars may considerably bias the mean flux level on occasions and therefore, the flux information should be considered with some caution.

CCD images were collected using the standard camera at the $1.2 \mathrm{~m}$ telescope. Most of the time the Johnson $B, V$, and $I$ filters were used. Depending on the CCD mounted, the field of view was either $7 \cdot 1^{\prime} \times 4.4^{\prime}(\mathrm{RCA})$ or $6.5^{\prime} \times 6.5^{\prime}$ (TK512). All CCD frames were corrected for bias and flat-field. These CCD images were mainly used as a guide for selecting potentially interesting candidates for spectroscopic observations and to provide differential astrometry and photometry with respect to Guide Star Catalogue entries (GSC; Lasker et al. 1990).

\section{Optical data analysis}

\subsection{Spectral classification}

We acquired medium resolution spectra for stars with spectral types earlier than $\approx \mathrm{M} 0$ and low resolution spectra for late $M$ type stars. Only a few bright stars having well documented spectral classification available in the literature were not observed.

Because of the relatively short wavelength range in the medium resolution mode $(\approx 500 \AA)$ computerized algorithms involving theoretical reference spectra are not suitable (Cayrel et al. 1991). Instead we extensively used the classification methods presented in Jaschek \& Jaschek (1987) and Turnsheck et al. (1985). Our spectral type determinations result from a visual comparison of our spectra with those of MK standards. For this purpose we used the library of Jaschek (1992); Turnsheck et al. (1985) and Jacoby et al. (1984). In addition we also observed few MK standard stars in the range G0 - M3 with the same instrumentation, wavelength range, and resolution as for our candidate stars.

Various classification criteria were considered depending on whether a blue medium resolution or a low resolution spectrum was available and depending on whether the star had an early or a late spectral type. For stars of spectral type earlier than $\mathrm{F}$, the classification depends on the strength of the CaII $\mathrm{H} \& \mathrm{~K}$ lines, which become much stronger than the hydrogen lines of the Balmer series. For spectral types later than G5 the CaI line ( $\lambda \quad 4227 \AA$ ) becomes highly sensitive to temperature. Metallic lines increase both in number and in intensity and are the main criteria of classification toward later spectral types. One of the major difficulties concerning the classification of our candidates is that for these active stars, a fraction of the energy is re-emitted in the Balmer lines. Therefore, all spectral determinations involving $\mathrm{H} \delta$ (a major indicator in our wavelength range) may undergo a shift of several tenths of spectral subtype. Whenever possible, the $\mathrm{H} \delta$ line has not been taken into account in the spectral type determination. We have also checked for luminosity effects using Sr II lines ( $\lambda 4077 \AA$ and $\lambda 4215 \AA)$ but no significant enhancement has been detected implying that most of our stars are main sequence stars. Although this is consistent with results from former Einstein stellar surveys (see e.g. Rosner et al. 1985), we cannot exclude that a small fraction of our candidates have erroneous luminosity classes. For most M stars only low resolution spectra were available and the main criteria of classification were the strength of the $\mathrm{CaOH}$ and $\mathrm{TiO}$ molecular bands.

Comparing our own spectral type determinations with those quoted in the literature for stars observed in various RGPS test areas or X-ray selected samples, we estimate that the uncertainty on the spectral classification should not exceed two spectral subtypes.

\subsection{Visual magnitudes}

Visual magnitudes were either extracted from the SIMBAD database or from the Guide Star Catalogue (Lasker et al. 1990). We also list the $B-V$ colour index in Tables 4 and 5 when available from the literature. For all stars having a spectral type determination we corrected the $V$ magnitudes taken from the GSC for colour effects according to relation (1) of Russell et al. (1990), assuming a $B-V$ colour index corresponding to the spectral type and neglecting interstellar reddening. The magnitudes of GSC stars without spectral types were left unchanged. In order to estimate the photometric quality of the corrected GSC magnitudes we compared these GSC based determinations with $V$ magnitudes extracted from SIMBAD header and associated literature. SIMBAD values generally arise from photometric measurements and have therefore much smaller associated errors than the photographic determinations used in the GSC. Based on 33 stars found in the course of RGPS optical identifications in various test fields and having both GSC and photometric SIMBAD measurements we conclude that ignoring a few pathological cases for which the difference in magnitudes is larger than $0.6 \mathrm{mag}$, the difference $\Delta m=m_{\mathrm{GSC}}-m_{\mathrm{SIMBAD}}$ had a mean value of 0.047 and a rms of 0.18 down to $m=13.5$. We thus adopted a $1 \sigma$ error of $0.2 \mathrm{mag}$ as statistically representative of the uncertainties affecting the colour corrected GSC $V$ magnitudes. For stars without spectral types we assumed a photometric error of $\approx 0.3 \mathrm{mag}$ (Russel et al. 1990). For the few stars with no SIMBAD nor GSC identification (generally $\mathrm{Me}$ stars fainter than $V \approx 14$ ) we derived visual magnitudes by differential photometry with nearby GSC stars using $V$ CCD images. The corresponding 
Table 1. Characteristics of the CCD used for spectroscopy and imagery

\begin{tabular}{lccrlc}
$\begin{array}{l}\text { CCD } \\
\text { name }\end{array}$ & Format & $\begin{array}{c}\text { Pixel size } \\
\text { in } \mu \mathrm{m}\end{array}$ & $\begin{array}{r}\text { Read out } \\
\text { noise }\left(\mathrm{e}^{-}\right)\end{array}$ & Instrument & $\begin{array}{c}\text { Scale } \\
\left({ }^{\prime \prime} / \text { pixel }\right)\end{array}$ \\
\hline RCA1 SID 501 & $323 \times 512$ & 30 & 110 & CARELEC & 1.22 \\
RCA3 SID 501EX & $323 \times 512$ & 30 & 55 & Imagery & 0.85 \\
THX1 TH 7882 & $384 \times 576$ & 23 & 11 & CARELEC & 0.94 \\
TK512 \# 1 TK512CB & $512 \times 512$ & 27 & 10 & CARELEC & 1.10 \\
TK512 \# 2 TK512CB & $512 \times 512$ & 27 & 9 & Imagery & 0.77 \\
\hline
\end{tabular}

photometric errors are dominated by those of the comparison GSC stars and are therefore of the order of $0.3 \mathrm{mag}$.

\subsection{Astrometry}

As for photometric data, the coordinates of the proposed optical counterparts were preferentially extracted from the SIMBAD database which most of the time lists high quality determinations (e.g. PPM). For the candidates without accurate SIMBAD positions we used the GSC coordinates. The coordinates of the remaining counterparts fainter than the GSC threshold were measured on CCD images with respect to nearby GSC stars. The maximum positional error for the overall identified set is therefore comparable to that internal to the GSC which is typically less than $2^{\prime \prime}$ (Russel et al. 1990; Egret et al. 1992). However, some high velocity objects may have a somewhat larger positional error since neither SIMBAD nor GSC coordinates are corrected for proper motion. In most cases, the error on the optical position is expected to be an order of magnitude smaller than the X-ray $90 \%$ confidence radius which is in the range of $17^{\prime \prime}$ to $58^{\prime \prime}$ (see Tables 2 and 3).

\subsection{Photometric correction of Ca II H\&K fluxes}

The CaII H\&K chromospheric emission lines are a very sensitive indicator of stellar activity. Studies based on Einstein X-ray data revealed that the luminosity of these lines correlate well with X-ray luminosity (e.g. Maggio et al. 1987). We extensively used this correlation in order to quantify the likelihood of our stellar X-ray identification (Guillout 1996; Motch et al. 1997). Ca II H\&K reemission fluxes were measured on the blue flux calibrated medium resolution spectra using MIDAS routines. The photospheric level was estimated by fitting Gaussian profiles to the broad wings of the absorption profiles. This allows a reasonably accurate measurement of the residual re-emission fluxes even in the cases of low chromospheric to photospheric contrast.

Although the blue medium resolution spectra are in principle flux calibrated, the Ca II H\&K fluxes extracted from these spectra have to be corrected for the unavoidable spectrophotometric errors resulting from unnoticed clouds, changing diffuse absorption in the UV or from erratic light losses in the spectrograph slit due to variable seeing conditions. For each candidate star we computed the $\lambda 4000 \AA$ to $\lambda 5500 \AA$ ( $V$ band) mean flux ratio corresponding to its spectral type. This flux ratio was estimated using digitized spectra extracted from the spectrophotometric library of Jacoby et al. (1984). The true mean flux at $\lambda 4000 \AA$ was then computed using as reference flux level the $V$ magnitude. Finally, the CaII H\&K emission lines fluxes were multiplied by the ratio of the expected $\lambda 4000 \AA$ flux to that measured from our "flux calibrated" medium resolution spectra. In all cases we assumed negligible interstellar absorption for these nearby sources (see next subsection).

\subsection{Stellar distances}

In the survey Cygnus region, most of the detected X-ray stars are closer than $300 \mathrm{pc}$ (Motch et al. 1997). At this distance, $\mathrm{A}_{\mathrm{V}}$ is always smaller than 0.5 over the whole investigated field (Neckel \& Klare 1980) and we decided not to apply additional photometric correction for interstellar absorption. Absolute magnitudes were derived from spectral type using the calibration of Schmidt-Kaler (1982). Taking into account the uncertainties on the $V$ magnitudes and absolute calibrations we estimate that the photometric distances have an error of the order of $20 \%$ yielding an additional $\approx 40 \%$ error on the X-ray and Ca II H\&K luminosities.

\section{Optical identifications}

The details of the procedure leading to positive optical identification are presented at large in Motch et al. (1997). Tables 4 and 5 summarize the status of the identification of each ROSAT source. Class acronyms are AC (active corona), AGN, CV (cataclysmic or possible precataclysmic systems), OB (normal OB star coronal emission), WD (isolated white dwarf) and ?? for unidentified source. For stars, the probability of the optical identification was estimated from the $\mathrm{Ca}$ II $\mathrm{H} \& \mathrm{~K}$ or $\mathrm{H} \alpha$ emission to $\mathrm{X}$-ray luminosity ratio, and/or from the probability of positional coincidence with a GSC star (Motch et al. 1997). We decided to accept as "secure" optical counterparts all 
proposed identifications having a probability larger than $98 \%$, implying a formal number of misidentified sources of the order of $\approx 1-2$ for the whole sample. For the sake of completeness, we also list in Table 4 and 5 the proposed counterparts having a probability of identification in the range $95 \%-98 \%$. These more uncertain cases are marked by a "?" as first letter of the identification name and the class acronym is followed by a "?".

We print in Fig. 1 and following for each source a summary of all available optical information. When we consider it necessary for follow-up optical observations, we display a finding chart based on one of our CCD images, with sometimes inserted a smaller field obtained in a different filter for comparison. In most cases, however, we print finding charts drawn from the Guide Star Catalogue (Lasker et al. 1990) extracted with the help of the STARCAT facility at ESO (Pirenne et al. 1993) and later from the SIMBAD catalogue GSC browser (PreiteMartinez \& Ochsenbein 1993).

When available, we also show medium and/or low resolution optical spectra of the proposed optical counterpart with inserted enlargement of the CaII H\&K lines when appropriate. When we fail to identify the source, we show the spectra obtained for all objects marked on the finding chart. Specific comments on individual sources are printed in Table 6.

Acknowledgements. We thank the night assistants at Observatoire de Haute-Provence for carrying out some of the observations at the $1.2 \mathrm{~m}$ telescope. The ROSAT project is supported by the Bundesministerium für Bildung, Wissenschaft, Forshung und Technologie (BMBF/DARA) and the MaxPlanck-Gesellschaft. C.M. acknowledges support from a CNRS-MPG cooperation contract and thanks Prof. J. Trümper and the ROSAT group for their hospitality and fruitful discussions. This research has made use of the SIMBAD database operated at CDS, Strasbourg, France. We thank B. Pirenne and F. Ochsenbein for providing easy access to the GSC. One finding chart was extracted from the Digitized Sky Survey, produced at the Space Telescope Science Institute (ST ScI) under U.S. Government grant NAG W-2166.

\section{References}

Banse K., Crane Ph., Ounnas Ch., Ponz D., 1983, "MIDAS" in Proc. of DECUS, Zurich, p. 87

Cash W., Charles P., Bowyer S., Walter F., Garmire G., Riegler G., 1980, ApJ 238, L71

Cayrel R., Perrin M.-N., Barbuy B., Buser R., 1991, A\&A 247, 108

Egret D., Halbwachs J.-L., Bässgen G., et al., 1992, in "Astronomy from Large Databases II", ESO Conf. and Work. Proc. 43, Heck A., Murtagh F. (eds.) p. 259

Guillout P., 1996, Ph.D. Thesis, Strasbourg, Université Louis Pasteur
Jacoby G.H., Hunter D.A., Christian C.A., 1984, ApJS 56, 257

Jaschek C., 1992, "Bibliothèque de standards MK" (private communication)

Jaschek C., Jaschek M., 1987, "The Classification of Stars", Press Syndicate of the University of Cambridge (U.S.A)

Lasker B.M., Sturch C.R., McLean B.J., Russel J.L., Jenkner H., Shara M.M., 1990, AJ 99, 2019

Lemaitre G., Kohler D., Lacroix D., Meunier J.P., Vin A., 1990, A\&A 228, 546

Maggio A., Sciortino S., Vaiana G.S., Majer P., Bookbinder J., Golub L., Harnden F.R., Rosner R., 1987, ApJ 315, 687

Motch C., Belloni T., Buckley D., Gottwald M., Hasinger G., Pakull M.W., Pietsch W., Reinsch K., Remillard R.A., Schmitt J.H.M.M., Trümper J., Zimmermann H.-U., 1991, A\&A 246, L24

Motch C., Guillout P., Haberl F., Pakull M.W., Pietsch W., Reinsch K., 1997, A\&A (in press)

Neckel Th., Klare G., 1980, A\&AS 42, 251

Nugent J.J., Jensen K.A., Nousek J.A., Garmire G.P., Mason K.O., Walter F.M., Bowyer C.S., Stern R.A., Riegler G.R., 1983, ApJS 51, 1

Pfeffermann E., Briel U.G., Hippmann H., Kettenring G., Metzner G., Predehl P., Reger G., Stephan K.H., Zombeck M.V., Chappell J., Murray S.S., 1986, SPIE 733, 519

Pirenne B., Albrecht M., Durand D., Gaudet S., 1993, in "Astronomical Data Analysis Software and Systems II", Hanish R.J., Brissenden R.J.V., Barnes J. (eds.) PASP 95, 99

Platais I., 1994, Bull. Inf. Centre Données Stellaires 44, 9

Preite-Martinez A., Ochsenbein F., 1993, in Proceedings of the "ESO/OAT Workshop on Handling and Archiving Data from Ground-based Telescopes" (May 1993) Pasian F. \& Albrecht M. (eds.)

Rosner R., Golub L., Vaiana G.S., 1985, ARA\&A 23, 413

Russel J.L., Lasker B.M., McLean B.J., Sturch C.R., Jenkner H., 1990, AJ 99, 2059

Schmidt-Kaler Th., 1982, in "Numerical Data and Functional Relationships in Science and Technology, Astronomy and Astrophysics", Vol 2b, Schaifers K. and Voigt H.H. (eds.) p. 17

Trümper J., 1983, Adv. Space. Res. 2, 241

Turnshek D.E., Turnshek D.A., Craine E.R., Boeshaar P.C., 1985, "An Atlas of Digital Spectra of Cool Stars", Western Research Company, Astronomy and Astrophysics Series, Vol. 1

Voges W., 1992, in Proceedings of Satellite Symposium 3, "International Space Year Conference", ESA ISY-3, 9

Voges W., Gruber R., Paul J., et al., 1992, in Proceedings of Satellite Symposium 3, "International Space Year Conference", ESA ISY-3, 1

Warwick R.S., Turner M.J.L., Watson M.G., Willingale R., 1985, Nat 317, 218

Wood K.S., Meekins J.F., Yentis D.J., et al., 1984, ApJS 56, 507 ,

Zimmermann H.U., Belloni T., Izzo C. Kahabka P., Schwentker O., 1992, MPE Report 48 
Table 2. X-ray characteristics of ROSAT survey sources detected with $M L \geq 8$ in the Cygnus test region. Entries are sorted by decreasing count rates

\begin{tabular}{|c|c|c|c|c|c|c|c|c|c|c|c|}
\hline $\begin{array}{c}\text { Source } \\
\text { index }\end{array}$ & $\begin{array}{c}\text { ROSAT } \\
\text { name }\end{array}$ & $\begin{array}{c}\text { Right } \\
\text { ascension }\end{array}$ & Declination & $\begin{array}{l}\mathrm{r}_{90} \\
\left({ }^{\prime \prime}\right)\end{array}$ & $\begin{array}{c}\text { Cnt rate } \\
\text { (cts/s) }\end{array}$ & $\begin{array}{l}\text { Error } \\
\text { (cts/s) }\end{array}$ & $\begin{array}{c}\text { Max } \\
\text { lik. }\end{array}$ & HR 1 & Error & HR 2 & Error \\
\hline 1 & RX J2112.7+5006 & $21 \mathrm{H} 12 \mathrm{M} 44.20 \mathrm{~S}$ & 50D06M16.9S & 17.5 & 1.310 & 0.041 & 3643.6 & -0.98 & 0.01 & 0.10 & 0.93 \\
\hline 2 & RX J2133.9+4535 & $21 \mathrm{H} 33 \mathrm{M} 58.78 \mathrm{~S}$ & 45D35M33.5S & 17.6 & 0.779 & 0.035 & 1297.3 & 0.05 & 0.05 & -0.17 & 0.07 \\
\hline 3 & RX J2052.7+4639 & $20 \mathrm{H} 52 \mathrm{M} 43.71 \mathrm{~S}$ & $46 \mathrm{D} 39 \mathrm{M} 41.2 \mathrm{~S}$ & 18.2 & 0.351 & 0.021 & 863.4 & -0.81 & 0.06 & -0.41 & 0.38 \\
\hline 4 & RX J2125.2+4942 & $21 \mathrm{H} 25 \mathrm{M} 12.37 \mathrm{~S}$ & $49 \mathrm{D} 42 \mathrm{M} 21.6 \mathrm{~S}$ & 18.2 & 0.250 & 0.019 & 422.2 & 0.08 & 0.09 & 0.32 & 0.11 \\
\hline 5 & RX J2100.8+4530 & $21 \mathrm{H} 00 \mathrm{M} 48.01 \mathrm{~S}$ & 45D30M09.1S & 18.5 & 0.244 & 0.017 & 425.1 & - & - & - & - \\
\hline 6 & RX J2106.0+5421 & $21 \mathrm{H} 06 \mathrm{M} 01.45 \mathrm{~S}$ & $54 \mathrm{D} 21 \mathrm{M} 56.5 \mathrm{~S}$ & 22.5 & 0.140 & 0.017 & 106.2 & -0.05 & 0.14 & -0.23 & 0.17 \\
\hline 7 & RX J2104.1+4912 & $21 \mathrm{H} 04 \mathrm{M} 10.04 \mathrm{~S}$ & $49 \mathrm{D} 12 \mathrm{M} 58.2 \mathrm{~S}$ & 17.9 & 0.139 & 0.014 & 267.3 & 0.14 & 0.11 & -0.14 & 0.14 \\
\hline 8 & RX J2130.8+4827 & $21 \mathrm{H} 30 \mathrm{M} 48.78 \mathrm{~S}$ & 48D27M39.0S & 19.3 & 0.136 & 0.015 & 168.7 & 0.04 & 0.12 & 0.17 & 0.16 \\
\hline 9 & RX J2120.9+4636 & $21 \mathrm{H} 20 \mathrm{M} 55.17 \mathrm{~S}$ & 46D36M18.0S & 19.0 & 0.114 & 0.013 & 150.5 & 0.31 & 0.12 & 0.07 & 0.14 \\
\hline 10 & RX J2124.7+4639 & $21 \mathrm{H} 24 \mathrm{M} 43.66 \mathrm{~S}$ & $46 \mathrm{D} 39 \mathrm{M} 52.1 \mathrm{~S}$ & 19.2 & 0.113 & 0.013 & 159.4 & 0.14 & 0.14 & -0.13 & 0.18 \\
\hline 11 & RX J2117.3+5044 & $21 \mathrm{H} 17 \mathrm{M} 19.42 \mathrm{~S}$ & 50D44M07.5S & 23.9 & 0.109 & 0.013 & 105.3 & -1.00 & 0.09 & - & - \\
\hline 12 & RX J2135.9+4728 & $21 \mathrm{H} 35 \mathrm{M} 54.38 \mathrm{~S}$ & $47 \mathrm{D} 28 \mathrm{M} 28.3 \mathrm{~S}$ & 18.8 & 0.101 & 0.012 & 160.4 & 0.87 & 0.12 & 0.76 & 0.11 \\
\hline 13 & RX J2102.6+4552 & $21 \mathrm{H} 02 \mathrm{M} 40.84 \mathrm{~S}$ & $45 \mathrm{D} 52 \mathrm{M} 57.4 \mathrm{~S}$ & 19.7 & 0.095 & 0.011 & 168.8 & - & - & - & - \\
\hline 14 & RX J2123.1+4831 & $21 \mathrm{H} 23 \mathrm{M} 08.11 \mathrm{~S}$ & $48 \mathrm{D} 31 \mathrm{M} 05.8 \mathrm{~S}$ & 21.1 & 0.077 & 0.011 & 81.2 & -0.43 & 0.18 & -0.40 & 0.40 \\
\hline 15 & RX J2055.3+5025 & $20 \mathrm{H} 55 \mathrm{M} 21.83 \mathrm{~S}$ & $50 \mathrm{D} 25 \mathrm{M} 33.3 \mathrm{~S}$ & 21.8 & 0.070 & 0.012 & 58.8 & - & - & - & - \\
\hline 16 & RX J2109.2+4810 & $21 \mathrm{H} 09 \mathrm{M} 17.12 \mathrm{~S}$ & 48D10M11.3S & 21.9 & 0.070 & 0.011 & 68.3 & -0.09 & 0.21 & 0.22 & 0.35 \\
\hline 17 & RX J2100.9+5103 & $21 \mathrm{H} 00 \mathrm{M} 59.30 \mathrm{~S}$ & 51D03M18.5S & 21.0 & 0.062 & 0.011 & 51.8 & -0.28 & 0.16 & 0.07 & 0.27 \\
\hline 18 & RX J2049.6+5119 & $20 \mathrm{H} 49 \mathrm{M} 37.35 \mathrm{~S}$ & 51D19M10.0S & 22.3 & 0.061 & 0.011 & 46.4 & - & - & - & - \\
\hline 19 & RX J2059.3+5303 & $20 \mathrm{H} 59 \mathrm{M} 20.66 \mathrm{~S}$ & 53D03M11.7S & 22.5 & 0.061 & 0.012 & 38.8 & -0.29 & 0.22 & -0.28 & 0.35 \\
\hline 20 & RX J2056.7+4940 & $20 \mathrm{H} 56 \mathrm{M} 42.63 \mathrm{~S}$ & 49D40M09.6S & 26.2 & 0.060 & 0.010 & 61.7 & - & - & - & - \\
\hline 21 & RX J2123.1+5021 & $21 \mathrm{H} 23 \mathrm{M} 11.94 \mathrm{~S}$ & $50 \mathrm{D} 21 \mathrm{M} 48.8 \mathrm{~S}$ & 22.7 & 0.059 & 0.010 & 52.2 & 0.13 & 0.18 & -0.52 & 0.21 \\
\hline 22 & RX J2107.8+4932 & $21 \mathrm{H} 07 \mathrm{M} 52.28 \mathrm{~S}$ & 49D32M $39.3 \mathrm{~S}$ & 23.4 & 0.049 & 0.009 & 44.6 & 0.21 & 0.21 & -0.09 & 0.24 \\
\hline 23 & RX J2100.1+4841 & $21 \mathrm{H} 00 \mathrm{M} 07.71 \mathrm{~S}$ & $48 \mathrm{D} 41 \mathrm{M} 28.6 \mathrm{~S}$ & 25.7 & 0.048 & 0.009 & 37.4 & - & - & - & - \\
\hline 24 & RX J2104.7+5223 & $21 \mathrm{H} 04 \mathrm{M} 43.36 \mathrm{~S}$ & $52 \mathrm{D} 23 \mathrm{M} 31.7 \mathrm{~S}$ & 25.2 & 0.048 & 0.011 & 33.0 & 0.47 & 0.42 & -0.11 & 0.27 \\
\hline 25 & RX J2044.6+4758 & $20 \mathrm{H} 44 \mathrm{M} 39.77 \mathrm{~S}$ & 47D58M01.9S & 21.0 & 0.048 & 0.009 & 49.6 & 0.09 & 0.40 & 0.93 & 0.84 \\
\hline 26 & RX J2107.3+5202 & $21 \mathrm{H} 07 \mathrm{M} 21.38 \mathrm{~S}$ & $52 \mathrm{D} 02 \mathrm{M} 57.1 \mathrm{~S}$ & 22.4 & 0.047 & 0.010 & 40.1 & - & - & - & - \\
\hline 27 & RX J2052.3+4820 & $20 \mathrm{H} 52 \mathrm{M} 23.88 \mathrm{~S}$ & 48D20M03.5S & 21.4 & 0.045 & 0.009 & 43.8 & - & - & - & - \\
\hline 28 & RX J2118.4+4356 & $21 \mathrm{H} 18 \mathrm{M} 25.99 \mathrm{~S}$ & $43 \mathrm{D} 56 \mathrm{M} 44.9 \mathrm{~S}$ & 19.8 & 0.045 & 0.009 & 48.6 & 1.00 & 0.30 & -0.43 & 0.21 \\
\hline 29 & RX J2109.3+5138 & $21 \mathrm{H} 09 \mathrm{M} 23.36 \mathrm{~S}$ & 51D38M05.5S & 27.7 & 0.041 & 0.009 & 29.7 & - & - & - & - \\
\hline 30 & RX J2120.4+4733 & $21 \mathrm{H} 20 \mathrm{M} 25.75 \mathrm{~S}$ & 47D33M42.8S & 24.5 & 0.040 & 0.008 & 34.9 & -0.52 & 0.21 & -0.57 & 0.62 \\
\hline 31 & RX J2104.2+5015 & $21 \mathrm{H} 04 \mathrm{M} 15.36 \mathrm{~S}$ & 50D15M08.3S & 57.5 & 0.038 & 0.008 & 16.0 & - & - & - & - \\
\hline 32 & RX J2057.3+4813 & $20 \mathrm{H} 57 \mathrm{M} 23.37 \mathrm{~S}$ & $48 \mathrm{D} 13 \mathrm{M} 29.2 \mathrm{~S}$ & 23.1 & 0.037 & 0.008 & 33.6 & - & - & - & - \\
\hline 33 & RX J2119.0+5207 & $21 \mathrm{H} 19 \mathrm{M} 00.28 \mathrm{~S}$ & $52 \mathrm{D} 07 \mathrm{M} 08.4 \mathrm{~S}$ & 22.5 & 0.036 & 0.009 & 32.3 & 0.13 & 0.45 & -0.26 & 0.50 \\
\hline 34 & RX J2117.8+5112 & $21 \mathrm{H} 17 \mathrm{M} 52.30 \mathrm{~S}$ & $51 \mathrm{D} 12 \mathrm{M} 08.4 \mathrm{~S}$ & 29.1 & 0.035 & 0.008 & 20.7 & 0.16 & 0.62 & 0.34 & 0.61 \\
\hline 35 & RX J2133.3+4726 & $21 \mathrm{H} 33 \mathrm{M} 22.11 \mathrm{~S}$ & $47 \mathrm{D} 26 \mathrm{M} 34.1 \mathrm{~S}$ & 22.3 & 0.035 & 0.008 & 41.8 & 0.96 & 0.27 & 0.62 & 0.16 \\
\hline 36 & RX J2134.0+4525 & $21 \mathrm{H} 34 \mathrm{M} 04.12 \mathrm{~S}$ & $45 \mathrm{D} 25 \mathrm{M} 25.3 \mathrm{~S}$ & 32.1 & 0.034 & 0.010 & 16.0 & 0.13 & 0.23 & -0.08 & 0.32 \\
\hline 37 & RX J2103.4+5021 & $21 \mathrm{H} 03 \mathrm{M} 25.45 \mathrm{~S}$ & $50 \mathrm{D} 21 \mathrm{M} 10.1 \mathrm{~S}$ & 20.5 & 0.034 & 0.008 & 35.0 & -0.01 & 0.28 & -0.40 & 0.36 \\
\hline 38 & RX J2040.6+4859 & $20 \mathrm{H} 40 \mathrm{M} 38.46 \mathrm{~S}$ & 48D59M56.6S & 20.9 & 0.033 & 0.008 & 38.4 & 0.40 & 0.94 & -0.55 & 0.91 \\
\hline 39 & RX J2102.9+4854 & $21 \mathrm{H} 02 \mathrm{M} 59.58 \mathrm{~S}$ & $48 \mathrm{D} 54 \mathrm{M} 42.4 \mathrm{~S}$ & 24.1 & 0.033 & 0.008 & 24.1 & 0.05 & 0.23 & -0.09 & 0.34 \\
\hline 40 & RX J2113.3+5140 & $21 \mathrm{H} 13 \mathrm{M} 23.70 \mathrm{~S}$ & 51D40M06.5S & 20.2 & 0.032 & 0.008 & 42.9 & - & - & - & - \\
\hline 41 & RX J2123.5+4621 & $21 \mathrm{H} 23 \mathrm{M} 34.16 \mathrm{~S}$ & $46 \mathrm{D} 21 \mathrm{M} 35.1 \mathrm{~S}$ & 23.3 & 0.032 & 0.008 & 28.1 & 0.45 & 0.22 & 0.08 & 0.25 \\
\hline 42 & RX J2100.9+4857 & $21 \mathrm{H} 00 \mathrm{M} 55.58 \mathrm{~S}$ & 48D57M08.0S & 26.3 & 0.032 & 0.008 & 23.5 & - & - & - & - \\
\hline 43 & RX J2130.1+4901 & $21 \mathrm{H} 30 \mathrm{M} 11.83 \mathrm{~S}$ & 49D01M33.0S & 22.2 & 0.032 & 0.008 & 26.6 & -0.09 & 0.23 & -0.62 & 0.38 \\
\hline 44 & RX J2054.1+4942 & $20 \mathrm{H} 54 \mathrm{M} 09.47 \mathrm{~S}$ & 49D42M47.0S & 31.5 & 0.032 & 0.009 & 14.8 & - & - & - & - \\
\hline 45 & RX J2116.6+4645 & $21 \mathrm{H} 16 \mathrm{M} 36.64 \mathrm{~S}$ & $46 \mathrm{D} 45 \mathrm{M} 27.8 \mathrm{~S}$ & 21.3 & 0.032 & 0.008 & 29.7 & 0.03 & 0.37 & -0.84 & 0.76 \\
\hline 46 & RX J2110.2+5333 & $21 \mathrm{H} 10 \mathrm{M} 16.55 \mathrm{~S}$ & 53D33M58.7S & 24.2 & 0.031 & 0.010 & 18.4 & -0.29 & 0.30 & -0.03 & 0.47 \\
\hline 47 & RX J2125.3+4642 & $21 \mathrm{H} 25 \mathrm{M} 20.50 \mathrm{~S}$ & $46 \mathrm{D} 42 \mathrm{M} 55.6 \mathrm{~S}$ & 25.9 & 0.031 & 0.008 & 26.0 & -0.35 & 0.38 & 0.32 & 0.83 \\
\hline 48 & RX J2118.5+5247 & $21 \mathrm{H} 18 \mathrm{M} 30.96 \mathrm{~S}$ & $52 \mathrm{D} 47 \mathrm{M} 45.4 \mathrm{~S}$ & 27.6 & 0.030 & 0.008 & 23.0 & -0.20 & 0.22 & -0.06 & 0.34 \\
\hline 49 & RX J2128.7+4409 & $21 \mathrm{H} 28 \mathrm{M} 46.26 \mathrm{~S}$ & 44D09M16.0S & 41.1 & 0.029 & 0.009 & 10.4 & 0.77 & 0.18 & -0.10 & 0.20 \\
\hline 50 & RX J2121.7+5049 & $21 \mathrm{H} 21 \mathrm{M} 46.06 \mathrm{~S}$ & $50 \mathrm{D} 49 \mathrm{M} 28.5 \mathrm{~S}$ & 26.5 & 0.029 & 0.008 & 28.0 & 0.10 & 0.23 & 0.07 & 0.27 \\
\hline 51 & RX J2132.5+4849 & $21 \mathrm{H} 32 \mathrm{M} 33.83 \mathrm{~S}$ & $48 \mathrm{D} 49 \mathrm{M} 41.3 \mathrm{~S}$ & 24.9 & 0.028 & 0.008 & 19.7 & -0.23 & 0.48 & 0.82 & 0.90 \\
\hline 52 & RX J2122.4+5023 & $21 \mathrm{H} 22 \mathrm{M} 29.15 \mathrm{~S}$ & 50D23M47.1S & 25.8 & 0.028 & 0.008 & 22.2 & 0.06 & 0.23 & 0.09 & 0.29 \\
\hline 53 & RX J2035.9+4900 & $20 \mathrm{H} 35 \mathrm{M} 57.61 \mathrm{~S}$ & 49D00M40.1S & 22.4 & 0.027 & 0.007 & 22.5 & 0.35 & 0.26 & -0.06 & 0.25 \\
\hline 54 & RX J2108.2+5313 & $21 \mathrm{H} 08 \mathrm{M} 17.65 \mathrm{~S}$ & $53 \mathrm{D} 13 \mathrm{M} 33.9 \mathrm{~S}$ & 27.5 & 0.027 & 0.009 & 15.2 & - & - & - & - \\
\hline 55 & RX J2128.6+4653 & $21 \mathrm{H} 28 \mathrm{M} 40.44 \mathrm{~S}$ & 46D53M55.3S & 22.9 & 0.026 & 0.007 & 21.7 & 0.14 & 0.39 & 0.02 & 0.54 \\
\hline 56 & RX J2048.0+4903 & $20 \mathrm{H} 48 \mathrm{M} 03.92 \mathrm{~S}$ & 49D03M08.1S & 20.8 & 0.026 & 0.007 & 27.5 & - & - & - & - \\
\hline 57 & RX J2115.4+4437 & $21 \mathrm{H} 15 \mathrm{M} 25.18 \mathrm{~S}$ & $44 \mathrm{D} 37 \mathrm{M} 24.6 \mathrm{~S}$ & 35.2 & 0.025 & 0.007 & 20.7 & 0.33 & 0.22 & 0.03 & 0.25 \\
\hline 58 & RX J2109.9+4809 & $21 \mathrm{H} 09 \mathrm{M} 55.86 \mathrm{~S}$ & 48D09M $20.1 \mathrm{~S}$ & 25.8 & 0.025 & 0.007 & 16.1 & - & - & - & - \\
\hline 59 & RX J2108.6+4927 & $21 \mathrm{H} 08 \mathrm{M} 37.97 \mathrm{~S}$ & 49D27M34.3S & 22.3 & 0.024 & 0.007 & 22.9 & 0.12 & 0.40 & 0.37 & 0.48 \\
\hline 60 & RX J2050.8+4743 & $20 \mathrm{H} 50 \mathrm{M} 48.56 \mathrm{~S}$ & 47D43M54.7S & 26.4 & 0.024 & 0.007 & 21.0 & 0.93 & 0.27 & 0.20 & 0.25 \\
\hline 61 & RX J2135.6+4523 & $21 \mathrm{H} 35 \mathrm{M} 41.85 \mathrm{~S}$ & $45 \mathrm{D} 23 \mathrm{M} 11.5 \mathrm{~S}$ & 24.9 & 0.023 & 0.008 & 10.6 & 0.37 & 0.25 & 0.53 & 0.29 \\
\hline 62 & RX J2119.9+5227 & $21 \mathrm{H} 19 \mathrm{M} 54.30 \mathrm{~S}$ & $52 \mathrm{D} 27 \mathrm{M} 36.4 \mathrm{~S}$ & 24.5 & 0.023 & 0.007 & 23.4 & 0.09 & 0.40 & 0.39 & 0.38 \\
\hline 63 & RX J2100.3+5219 & $21 \mathrm{H} 00 \mathrm{M} 19.66 \mathrm{~S}$ & 52D19M04.9S & 25.2 & 0.022 & 0.008 & 19.0 & 0.16 & 0.28 & -0.05 & 0.28 \\
\hline
\end{tabular}




\begin{tabular}{|c|c|c|c|c|c|c|c|c|c|c|c|}
\hline $\begin{array}{c}\text { Source } \\
\text { index }\end{array}$ & $\begin{array}{c}\text { ROSAT } \\
\text { name }\end{array}$ & $\begin{array}{c}\text { Right } \\
\text { ascension }\end{array}$ & Declination & $\begin{array}{l}\mathrm{r}_{90} \\
\left({ }^{\prime \prime}\right)\end{array}$ & $\begin{array}{c}\text { Cnt rate } \\
(\mathrm{cts} / \mathrm{s})\end{array}$ & $\begin{array}{l}\text { Error } \\
\text { (cts/s) }\end{array}$ & $\begin{array}{c}\text { Max } \\
\text { lik. }\end{array}$ & HR 1 & Error & HR 2 & Error \\
\hline 64 & RX J2056.3+4817 & $20 \mathrm{H} 56 \mathrm{M} 23.14 \mathrm{~S}$ & $48 \mathrm{D} 17 \mathrm{M} 22.5 \mathrm{~S}$ & 28.5 & 0.022 & 0.007 & 13.5 & 0.54 & 0.27 & -0.08 & 0.24 \\
\hline 66 & RX J2041.9+4746 & $20 \mathrm{H} 41 \mathrm{M} 57.45 \mathrm{~S}$ & 47D46M08.8S & 43.5 & 0.020 & 0.007 & 8.4 & - & - & - & - \\
\hline 67 & RX J2126.3+4654 & $21 \mathrm{H} 26 \mathrm{M} 23.47 \mathrm{~S}$ & 46D54M31.0S & 32.8 & 0.020 & 0.007 & 10.2 & - & - & - & - \\
\hline 68 & RX J2055.8+5044 & 20H55M51.81S & 50D44M32.3S & 28.0 & 0.019 & 0.007 & 15.1 & - & - & - & - \\
\hline 69 & RX J2117.5+5127 & $21 \mathrm{H} 17 \mathrm{M} 33.20 \mathrm{~S}$ & $51 \mathrm{D} 27 \mathrm{M} 10.2 \mathrm{~S}$ & 23.8 & 0.019 & 0.007 & 12.8 & -0.52 & 0.28 & -0.71 & 0.79 \\
\hline 71 & RX J2131.2+4533 & $21 \mathrm{H} 31 \mathrm{M} 17.47 \mathrm{~S}$ & 45D33M14.4S & 25.4 & 0.018 & 0.007 & 9.3 & - & - & - & - \\
\hline 72 & RX J2054.6+5120 & $20 \mathrm{H} 54 \mathrm{M} 41.48 \mathrm{~S}$ & 51D20M38.7S & 45.2 & 0.018 & 0.006 & 12.0 & - & - & - & - \\
\hline 73 & RX J2121.5+4317 & $21 \mathrm{H} 21 \mathrm{M} 35.61 \mathrm{~S}$ & 43D17M29.4S & 57.6 & 0.018 & 0.006 & 8.2 & - & - & - & - \\
\hline 74 & RX J2128.4+4900 & $21 \mathrm{H} 28 \mathrm{M} 24.79 \mathrm{~S}$ & 49D00M39.3S & 29.4 & 0.017 & 0.006 & 10.5 & - & - & - & - \\
\hline 75 & RX J2058.5+4836 & $20 \mathrm{H} 58 \mathrm{M} 31.16 \mathrm{~S}$ & 48D36M01.5S & 30.1 & 0.017 & 0.006 & 10.7 & - & - & - & - \\
\hline 76 & RX J2130.0+4740 & 21H30M05.91S & 47D40M30.8S & 24.9 & 0.017 & 0.006 & 12.6 & - & - & - & - \\
\hline 77 & RX J2106.2+4437 & 21H06M17.37S & $44 \mathrm{D} 37 \mathrm{M} 00.9 \mathrm{~S}$ & 21.2 & 0.017 & 0.006 & 16.7 & 0.01 & 0.44 & 0.21 & 0.66 \\
\hline 78 & RX J2128.5+4626 & $21 \mathrm{H} 28 \mathrm{M} 30.05 \mathrm{~S}$ & 46D26M55.3S & 32.4 & 0.017 & 0.007 & 11.1 & - & - & - & - \\
\hline 81 & RX J2046.7+4728 & $20 \mathrm{H} 46 \mathrm{M} 45.18 \mathrm{~S}$ & 47D28M34.7S & 34.1 & 0.017 & 0.006 & 9.2 & - & - & - & - \\
\hline 82 & RX J2136.6+4911 & 21H36M39.63S & 49D11M00.9S & 23.5 & 0.017 & 0.006 & 19.0 & - & - & - & - \\
\hline 83 & RX J2118.6+5010 & $21 \mathrm{H} 18 \mathrm{M} 41.25 \mathrm{~S}$ & 50D10M53.8S & 24.9 & 0.017 & 0.005 & 19.1 & 0.74 & 0.74 & 0.12 & 0.43 \\
\hline 84 & RX J2130.3+4709 & 21H30M20.75S & 47D09M52.9S & 26.1 & 0.016 & 0.006 & 10.6 & -0.14 & 0.26 & -0.26 & 0.43 \\
\hline 85 & RX J2116.0+4827 & 21H16M03.35S & 48D27M00.3S & 33.4 & 0.016 & 0.006 & 8.9 & -0.60 & 0.60 & -0.74 & 2.32 \\
\hline 86 & RX J2124.7+4714 & $21 \mathrm{H} 24 \mathrm{M} 42.93 \mathrm{~S}$ & 47D14M36.5S & 24.6 & 0.016 & 0.006 & 12.2 & - & - & - & - \\
\hline 87 & RX J2117.5+4330 & $21 \mathrm{H} 17 \mathrm{M} 32.58 \mathrm{~S}$ & 43D30M59.6S & 33.3 & 0.016 & 0.006 & 8.7 & - & - & - & - \\
\hline 88 & RX J2137.6+4916 & $21 \mathrm{H} 37 \mathrm{M} 38.54 \mathrm{~S}$ & 49D16M09.7S & 26.3 & 0.016 & 0.005 & 15.3 & - & - & - & - \\
\hline 89 & RX J2100.6+5039 & $21 \mathrm{H} 00 \mathrm{M} 38.19 \mathrm{~S}$ & 50D39M27.0S & 29.4 & 0.016 & 0.006 & 9.1 & - & - & - & - \\
\hline 90 & RX J2110.5+4913 & $21 \mathrm{H} 10 \mathrm{M} 32.18 \mathrm{~S}$ & 49D13M32.9S & 29.5 & 0.016 & 0.006 & 8.6 & - & - & - & - \\
\hline 91 & RX J2121.5+4732 & 21H21M30.99S & 47D32M52.1S & 31.2 & 0.016 & 0.006 & 8.4 & 0.08 & 0.24 & 0.55 & 0.27 \\
\hline 92 & RX J2052.8+4723 & $20 \mathrm{H} 52 \mathrm{M} 53.05 \mathrm{~S}$ & $47 \mathrm{D} 23 \mathrm{M} 34.6 \mathrm{~S}$ & 32.4 & 0.015 & 0.006 & 8.0 & - & - & - & - \\
\hline 93 & RX J2118.6+5039 & $21 \mathrm{H} 18 \mathrm{M} 40.10 \mathrm{~S}$ & 50D39M06.4S & 35.3 & 0.015 & 0.006 & 8.3 & - & - & - & - \\
\hline 94 & RX J2122.3+4730 & $21 \mathrm{H} 22 \mathrm{M} 20.15 \mathrm{~S}$ & 47D30M45.4S & 26.1 & 0.015 & 0.006 & 10.4 & -0.22 & 0.28 & -0.35 & 0.48 \\
\hline 95 & RX J2050.4+4913 & $20 \mathrm{H} 50 \mathrm{M} 27.45 \mathrm{~S}$ & 49D13M16.2S & 25.6 & 0.015 & 0.006 & 9.3 & - & - & - & - \\
\hline 96 & RX J2113.0+4834 & $21 \mathrm{H} 13 \mathrm{M} 05.12 \mathrm{~S}$ & 48D34M30.5S & 29.0 & 0.015 & 0.006 & 9.9 & 0.28 & 0.42 & -0.07 & 0.46 \\
\hline 97 & RX J2134.2+4911 & $21 \mathrm{H} 34 \mathrm{M} 15.78 \mathrm{~S}$ & 49D11M23.0S & 21.7 & 0.015 & 0.005 & 21.7 & - & - & - & - \\
\hline 100 & RX J2123.2+5057 & $21 \mathrm{H} 23 \mathrm{M} 14.80 \mathrm{~S}$ & 50D57M09.5S & 40.6 & 0.014 & 0.005 & 10.3 & - & - & - & - \\
\hline 101 & RX J2053.4+4759 & $20 \mathrm{H} 53 \mathrm{M} 28.87 \mathrm{~S}$ & 47D59M14.3S & 26.2 & 0.014 & 0.006 & 9.0 & - & - & - & - \\
\hline 102 & RX J2124.3+5059 & $21 \mathrm{H} 24 \mathrm{M} 18.31 \mathrm{~S}$ & $50 \mathrm{D} 59 \mathrm{M} 52.4 \mathrm{~S}$ & 24.4 & 0.013 & 0.005 & 17.3 & 0.33 & 0.29 & 0.60 & 0.23 \\
\hline 103 & RX J2114.1+4840 & 21H14M09.88S & 48D40M27.9S & 28.6 & 0.013 & 0.005 & 14.4 & - & - & - & - \\
\hline 104 & RX J2043.7+4727 & $20 \mathrm{H} 43 \mathrm{M} 44.64 \mathrm{~S}$ & 47D27M11.9S & 28.0 & 0.013 & 0.006 & 8.8 & - & - & - & - \\
\hline 105 & RX J2122.6+4956 & $21 \mathrm{H} 22 \mathrm{M} 40.00 \mathrm{~S}$ & 49D56M04.0S & 29.8 & 0.013 & 0.005 & 11.7 & 0.29 & 0.46 & 0.41 & 0.43 \\
\hline 106 & RX J2103.6+4845 & $21 \mathrm{H} 03 \mathrm{M} 39.94 \mathrm{~S}$ & $48 \mathrm{D} 45 \mathrm{M} 41.1 \mathrm{~S}$ & 26.9 & 0.013 & 0.005 & 9.7 & - & - & - & - \\
\hline 107 & RX J2059.8+4937 & 20H59M48.59S & 49D37M34.6S & 36.2 & 0.013 & 0.005 & 8.8 & - & - & - & - \\
\hline 108 & RX J2126.9+4636 & $21 \mathrm{H} 26 \mathrm{M} 58.87 \mathrm{~S}$ & 46D36M43.9S & 33.7 & 0.013 & 0.005 & 8.1 & - & - & - & - \\
\hline 109 & RX J $2120.2+5127$ & $21 \mathrm{H} 20 \mathrm{M} 17.48 \mathrm{~S}$ & 51D27M46.7S & 25.2 & 0.013 & 0.006 & 12.1 & - & - & - & - \\
\hline 110 & RX J2130.7+4919 & $21 \mathrm{H} 30 \mathrm{M} 44.80 \mathrm{~S}$ & 49D19M19.2S & 26.2 & 0.012 & 0.005 & 11.7 & -0.18 & 0.35 & -1.00 & 0.70 \\
\hline 111 & RX J2123.7+4636 & $21 \mathrm{H} 23 \mathrm{M} 44.89 \mathrm{~S}$ & 46D36M59.4S & 30.9 & 0.012 & 0.005 & 8.7 & - & - & - & - \\
\hline 112 & RX J2043.4+5002 & $20 \mathrm{H} 43 \mathrm{M} 27.86 \mathrm{~S}$ & $50 \mathrm{D} 02 \mathrm{M} 33.9 \mathrm{~S}$ & 28.4 & 0.012 & 0.005 & 8.2 & - & - & - & - \\
\hline 113 & RX J2107.6+5048 & $21 \mathrm{H} 07 \mathrm{M} 38.56 \mathrm{~S}$ & $50 \mathrm{D} 48 \mathrm{M} 51.5 \mathrm{~S}$ & 23.8 & 0.012 & 0.005 & 12.9 & - & - & - & - \\
\hline 114 & RX J2117.5+5243 & $21 \mathrm{H} 17 \mathrm{M} 31.42 \mathrm{~S}$ & $52 \mathrm{D} 43 \mathrm{M} 09.5 \mathrm{~S}$ & 22.9 & 0.012 & 0.004 & 18.3 & - & - & - & - \\
\hline 115 & RX J $2120.8+5209$ & $21 \mathrm{H} 20 \mathrm{M} 49.63 \mathrm{~S}$ & $52 \mathrm{D} 09 \mathrm{M} 27.7 \mathrm{~S}$ & 25.8 & 0.011 & 0.005 & 12.3 & - & - & - & - \\
\hline 116 & RX J2121.8+5135 & $21 \mathrm{H} 21 \mathrm{M} 51.46 \mathrm{~S}$ & 51D35M16.5S & 27.9 & 0.010 & 0.005 & 9.2 & - & - & - & - \\
\hline 117 & RX J2121.0+5049 & $21 \mathrm{H} 21 \mathrm{M} 02.19 \mathrm{~S}$ & 50D49M57.6S & 28.9 & 0.010 & 0.004 & 10.4 & - & - & - & - \\
\hline 118 & RX J2058.1+4552 & $20 \mathrm{H} 58 \mathrm{M} 11.60 \mathrm{~S}$ & 45D52M57.6S & 24.4 & 0.010 & 0.005 & 9.4 & - & - & - & - \\
\hline 119 & RX J2112.9+5314 & $21 \mathrm{H} 12 \mathrm{M} 55.73 \mathrm{~S}$ & 53D14M24.0S & 34.5 & 0.010 & 0.005 & 8.8 & - & - & - & - \\
\hline 120 & RX J2122.6+4856 & $21 \mathrm{H} 22 \mathrm{M} 37.14 \mathrm{~S}$ & $48 \mathrm{D} 56 \mathrm{M} 13.8 \mathrm{~S}$ & 30.1 & 0.009 & 0.004 & 8.1 & - & - & - & - \\
\hline 121 & RX J2122.0+5059 & $21 \mathrm{H} 22 \mathrm{M} 02.40 \mathrm{~S}$ & 50D59M18.7S & 29.9 & 0.009 & 0.004 & 9.0 & - & - & - & - \\
\hline 122 & RX J2105.7+4933 & 21H05M45.89S & 49D33M36.5S & 32.6 & 0.009 & 0.004 & 8.0 & - & - & - & - \\
\hline 123 & RX J2116.0+5254 & 21H16M03.91S & 52D54M56.1S & 27.7 & 0.009 & 0.004 & 8.3 & - & - & - & - \\
\hline 124 & RX J2059.6+4731 & $20 \mathrm{H} 59 \mathrm{M} 40.20 \mathrm{~S}$ & 47D31M11.8S & 23.4 & 0.008 & 0.004 & 8.4 & - & - & - & - \\
\hline 125 & RX J2100.8+5213 & 21H00M52.03S & 52D13M58.1S & 31.1 & 0.008 & 0.004 & 8.9 & - & - & - & - \\
\hline 126 & RX J2111.8+4942 & $21 \mathrm{H} 11 \mathrm{M} 53.70 \mathrm{~S}$ & 49D42M33.3S & 25.9 & 0.008 & 0.004 & 9.2 & - & - & - & - \\
\hline 127 & RX J2108.9+4958 & 21H08M56.32S & 49D58M07.8S & 30.8 & 0.008 & 0.004 & 8.1 & - & - & - & - \\
\hline 128 & RX J2122.9+4941 & $21 \mathrm{H} 22 \mathrm{M} 58.94 \mathrm{~S}$ & 49D41M00.9S & 23.7 & 0.006 & 0.003 & 9.8 & - & - & - & - \\
\hline
\end{tabular}


Table 3. X-ray characteristics of ROSAT survey sources detected with $7 \leq M L<8$ in the Cygnus test region. Entries are sorted by decreasing count rates

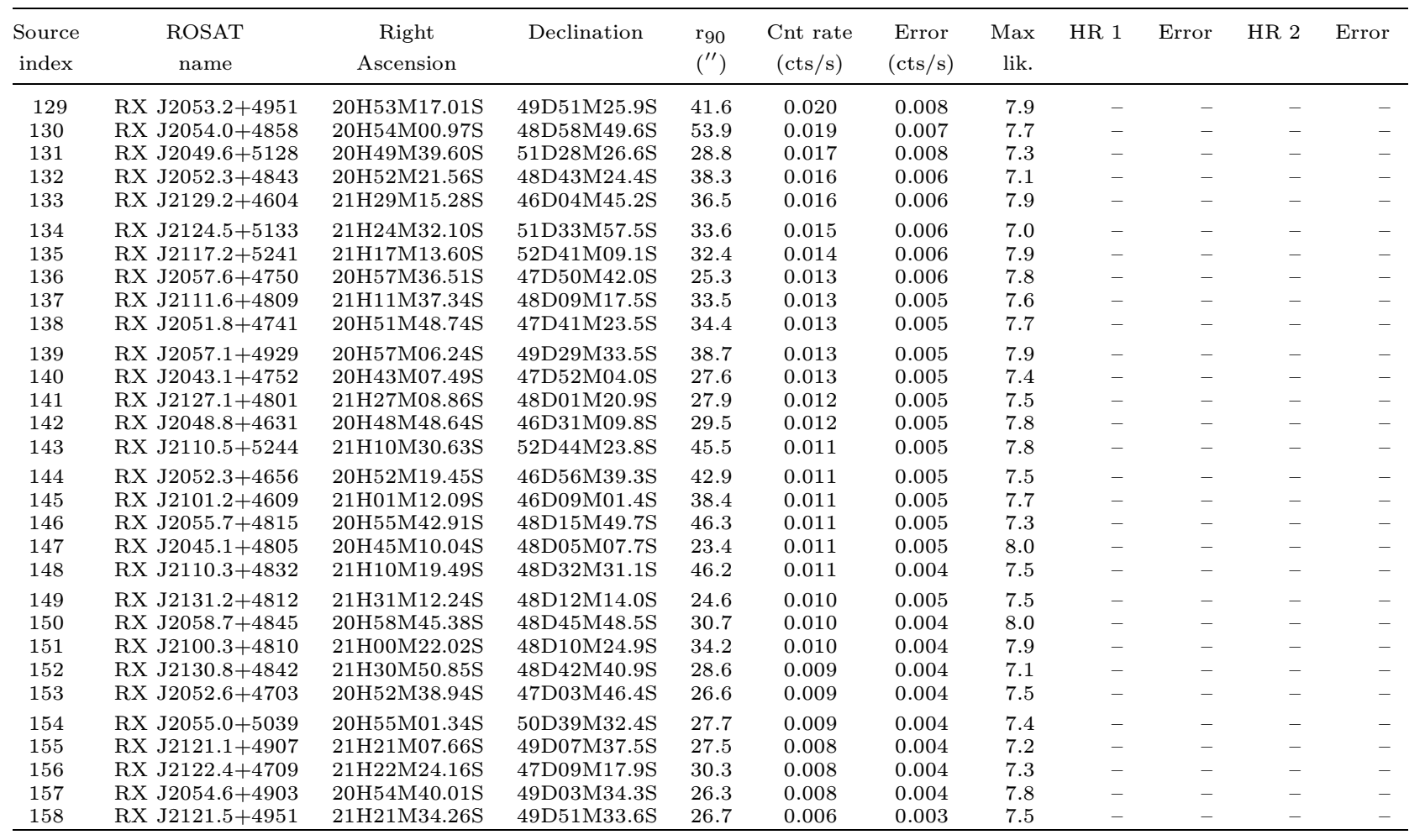


Table 4. Optical properties of all proposed optical counterparts in the Cygnus test region for sources with $M L \geq 8$. The last column lists the distance between X-ray an optical position in units of the $90 \%$ confidence radius. For the sake of completeness, we also list the proposed counterparts having a probability of identification in the range 95\% - 98\%. These more uncertain cases are marked by a "?" as first letter of the identification name and the class acronym is followed by a "?"

\begin{tabular}{|c|c|c|c|c|c|c|c|c|c|}
\hline $\begin{array}{l}\text { Source } \\
\text { index }\end{array}$ & $\begin{array}{c}\text { ROSAT } \\
\text { name }\end{array}$ & Class & Identification & $\begin{array}{l}\text { Spectral } \\
\text { type }\end{array}$ & V & $B-V$ & $\begin{array}{c}\text { RA } \\
\text { (optical) }\end{array}$ & $\begin{array}{c}\text { DEC } \\
\text { (optical) }\end{array}$ & $\begin{array}{r}D_{\mathrm{X}-\mathrm{Opt}} \\
\left(\mathrm{r}_{90}\right)\end{array}$ \\
\hline 1 & RX J2112.7+5006 & WD & GD 394 & DAw & 13.09 & -0.24 & $21 \mathrm{H} 12 \mathrm{M} 43.50 \mathrm{~S}$ & 50D06M17.0S & .38 \\
\hline 2 & RX J2133.9+4535 & $\mathrm{AC}$ & HR 8252 & G8III & 4.00 & 0.88 & $21 \mathrm{H} 33 \mathrm{M} 58.87 \mathrm{~S}$ & 45D $35 \mathrm{M} 35.3 \mathrm{~S}$ & .12 \\
\hline 3 & RX J2052.7+4639 & WD & $\mathrm{C}$ & DO & 17.50 & - & $20 \mathrm{H} 52 \mathrm{M} 43.87 \mathrm{~S}$ & $46 \mathrm{D} 39 \mathrm{M} 35.2 \mathrm{~S}$ & .35 \\
\hline 4 & RX J2125.2+4942 & $\mathrm{AC}$ & $\mathrm{A}=\mathrm{GSC}-0359801594$ & $\mathrm{~K} 4 \mathrm{~V}$ & 10.95 & - & $21 \mathrm{H} 25 \mathrm{M} 12.11 \mathrm{~S}$ & $49 \mathrm{D} 42 \mathrm{M} 20.3 \mathrm{~S}$ & .15 \\
\hline 5 & RX J2100.8+4530 & $\mathrm{AC}$ & SAO 50350 & G3V & 8.82 & 0.72 & $21 \mathrm{H} 00 \mathrm{M} 46.72 \mathrm{~S}$ & 45D30M09.6S & .73 \\
\hline 6 & RX J2106.0+5421 & $\mathrm{AC}$ & HD 235440 & F $8 \mathrm{~V}$ & 9.20 & 0.70 & $21 \mathrm{H} 06 \mathrm{M} 00.57 \mathrm{~S}$ & $54 \mathrm{D} 21 \mathrm{M} 51.0 \mathrm{~S}$ & .42 \\
\hline 7 & RX J2104.1+4912 & $\mathrm{AC}$ & $\mathrm{B}$ & M4e & 16.00 & - & $21 \mathrm{H} 04 \mathrm{M} 10.64 \mathrm{~S}$ & 49D12M47.5S & .69 \\
\hline 8 & RX J2130.8+4827 & $\mathrm{AC}$ & SAO 50961 & G3V & 8.54 & 0.74 & $21 \mathrm{H} 30 \mathrm{M} 47.88 \mathrm{~S}$ & $48 \mathrm{D} 27 \mathrm{M} 33.0 \mathrm{~S}$ & .56 \\
\hline 9 & RX J2120.9+4636 & $\mathrm{AC}$ & $\mathrm{A}=\mathrm{GSC}-0358903858$ & F9V & 9.73 & - & $21 \mathrm{H} 20 \mathrm{M} 55.39 \mathrm{~S}$ & $46 \mathrm{D} 36 \mathrm{M} 13.0 \mathrm{~S}$ & .29 \\
\hline 10 & RX J2124.7+4639 & $\mathrm{AC}$ & $\mathrm{A}+\mathrm{B}$ & $\mathrm{K} 3+\mathrm{K} 7 \mathrm{~V}$ & 10.59 & - & $21 \mathrm{H} 24 \mathrm{M} 43.75 \mathrm{~S}$ & $46 \mathrm{D} 39 \mathrm{M} 42.2 \mathrm{~S}$ & .52 \\
\hline 11 & RX J2117.3+5044 & WD & $\mathrm{B}=\mathrm{LAN} 121$ & DA & 13.39 & - & $21 \mathrm{H} 17 \mathrm{M} 17.72 \mathrm{~S}$ & 50D44M06.0S & .67 \\
\hline 12 & RX J2135.9+4728 & AGN & $\mathrm{B}$ & Seyf 1 & 16.30 & - & $21 \mathrm{H} 35 \mathrm{M} 53.97 \mathrm{~S}$ & 47D28M20.9S & .45 \\
\hline 13 & RX J2102.6+4552 & $\mathrm{AC}$ & HD 200560 & K3V & 7.68 & 0.97 & $21 \mathrm{H} 02 \mathrm{M} 38.67 \mathrm{~S}$ & $45 \mathrm{D} 52 \mathrm{M} 58.4 \mathrm{~S}$ & 1.14 \\
\hline 14 & RX J2123.1+4831 & $\mathrm{AC}$ & HD 203839 & F0V & 7.80 & 0.40 & $21 \mathrm{H} 23 \mathrm{M} 07.70 \mathrm{~S}$ & 48D31M09.3S & .25 \\
\hline 15 & RX J2055.3+5025 & $\mathrm{AC}$ & $\mathrm{A}=\mathrm{GSC}-0358301038$ & G0V & 9.58 & - & $20 \mathrm{H} 55 \mathrm{M} 23.35 \mathrm{~S}$ & $50 \mathrm{D} 25 \mathrm{M} 36.4 \mathrm{~S}$ & .69 \\
\hline 16 & RX J2109.2+4810 & $\mathrm{AC}$ & $\mathrm{A}=\mathrm{GSC}-0359205781$ & $\mathrm{~K} 7 \mathrm{~V}$ & 11.40 & - & $21 \mathrm{H} 09 \mathrm{M} 17.84 \mathrm{~S}$ & $48 \mathrm{D} 10 \mathrm{M} 12.8 \mathrm{~S}$ & .34 \\
\hline 17 & RX J2100.9+5103 & $\mathrm{AC}$ & $\mathrm{A}=\mathrm{G} 231-24$ & $\mathrm{M} 2 \mathrm{Ve}$ & 14.80 & - & $21 \mathrm{H} 00 \mathrm{M} 59.62 \mathrm{~S}$ & 51D03M15.0S & .23 \\
\hline 18 & RX J2049.6+5119 & $\mathrm{AC}$ & HD 198638 & $\mathrm{~F} 2 \mathrm{~V}$ & 6.70 & 0.50 & $20 \mathrm{H} 49 \mathrm{M} 36.46 \mathrm{~S}$ & 51D18M54.2S & .80 \\
\hline 19 & RX J2059.3+5303 & $\mathrm{AC}$ & $\mathrm{A}=\mathrm{GSC}-0395$ & $\mathrm{M} 3.5 \mathrm{Ve}$ & 14.10 & - & 20H59M20.09S & 53D03M04.9S & .37 \\
\hline 20 & RX J2056.7+4940 & $\mathrm{AC}$ & SAO 50269 & A7V & 9.90 & 0.10 & $20 \mathrm{H} 56 \mathrm{M} 41.86 \mathrm{~S}$ & 49D40M17.8S & .42 \\
\hline 21 & RX J2123.1+5021 & $\mathrm{AC}$ & $\mathrm{A}=\mathrm{BD}+493512$ & G0V & 9.56 & - & $21 \mathrm{H} 23 \mathrm{M} 12.30 \mathrm{~S}$ & $50 \mathrm{D} 21 \mathrm{M} 47.2 \mathrm{~S}$ & .17 \\
\hline 22 & RX J2107.8+4932 & $\mathrm{AC}$ & $\mathrm{A}=\mathrm{GSC}-0359600261$ & G8V & 10.66 & - & $21 \mathrm{H} 07 \mathrm{I}$ & 49D32 & .50 \\
\hline 23 & RX J2100.1+4841 & $\mathrm{AC}$ & $\mathrm{BD}+483260 \mathrm{E}$ & G9V & 12.03 & - & $21 \mathrm{H} 00 \mathrm{M} 07.88 \mathrm{~S}$ & $48 \mathrm{D} 41 \mathrm{M} 29.6 \mathrm{~S}$ & .08 \\
\hline 24 & RX J2104.7+5223 & $\mathrm{AC}$ & $\mathrm{A}=\mathrm{GSC}-0360000513$ & $\mathrm{G} 2 \mathrm{~V}$ & 11.44 & - & $21 \mathrm{H} 04 \mathrm{M} 44.32 \mathrm{~S}$ & 52D $23 \mathrm{M} 25.8 \mathrm{~S}$ & .42 \\
\hline 25 & RX J2044.6+4758 & $\mathrm{AC}$ & $\mathrm{A}=\mathrm{GSC}-0357800872$ & & 11.43 & - & $20 \mathrm{H} 44 \mathrm{M} 37.95 \mathrm{~S}$ & 47D58M08.3S & .91 \\
\hline 26 & RX J2107.3+5202 & $\mathrm{AC}$ & V1061 Cyg $=$ HD 235444 & $\mathrm{~F} \varepsilon$ & 9.24 & 0.55 & $21 \mathrm{H} 07 \mathrm{M} 20.33 \mathrm{~S}$ & $52 \mathrm{D} 02 \mathrm{M} 56.3 \mathrm{~S}$ & .42 \\
\hline 27 & RX J2052.3+4820 & AC & $\mathrm{A}=$ GSC-0357901469 & G9V & 11.64 & 0.00 & $20 \mathrm{H} 52 \mathrm{M} 23.08 \mathrm{~S}$ & $48 \mathrm{D} 19 \mathrm{~N}$ & .47 \\
\hline 28 & RX J2118.4+4356 & $\mathrm{OB}$ & HR 8154 & $\mathrm{O} 8 \mathrm{Ve}$ & 5.01 & .03 & $21 \mathrm{H} 18 \mathrm{M} 27.05 \mathrm{~S}$ & 43D56M $45.8 \mathrm{~S}$ & .59 \\
\hline 29 & RX J2109.3+5138 & $\mathrm{AC}$ & $\mathrm{A}=\mathrm{GSC}-0360000175$ & G3V & 9.66 & - & $21 \mathrm{H} 09 \mathrm{M} 22.23 \mathrm{~S}$ & 51D37M50.2S & .67 \\
\hline 30 & RX J2120.4+4733 & $\mathrm{AC}$ & HD 203418 & $\mathrm{~K} 4 \mathrm{~V}$ & 8.30 & 1.60 & $21 \mathrm{H} 20 \mathrm{M} 25.27 \mathrm{~S}$ & 47D33M35.0S & .37 \\
\hline 31 & RX J2104.2+5015 & $\mathrm{AC}$ & A & $\mathrm{K} 7 \mathrm{e}$ & 15.00 & - & $\mathrm{N} / \mathrm{A}$ & $\mathrm{N} / \mathrm{A}$ & - \\
\hline 32 & $3+4813$ & $\mathrm{AC}$ & $\mathrm{A}=\mathrm{C}$ & $\mathrm{A} 2 \mathrm{~m}$ & 10.93 & - & $20 \mathrm{H} 57 \mathrm{M} 22$ & 48D13M3 & .36 \\
\hline 33 & RX J2119.0+5207 & $\mathrm{AC}$ & $\mathrm{A}=\mathrm{GSC}-0360101235$ & G1V & 11.72 & - & $21 \mathrm{H} 18 \mathrm{M} 59.28 \mathrm{~S}$ & 52D07M03.3S & .46 \\
\hline 34 & RX J2117.8+5112 & $\mathrm{AC}$ & HD 203028 & $\mathrm{~A} 2 \mathrm{~V}$ & 8.36 & 0.23 & $21 \mathrm{H} 17 \mathrm{M} 52.51 \mathrm{~S}$ & 51D12M25.7S & .60 \\
\hline 35 & RX J2133.3+4726 & ?? & & & & - & & & \\
\hline 36 & RX J2134.0+4525 & $\mathrm{AC}$ & $\mathrm{A}=\mathrm{GSC}-0$ & G3V & 11.07 & - & $21 \mathrm{H} 34 \mathrm{~N}$ & 45D25M33.6S & .52 \\
\hline 37 & RX J2103.4+5021 & $\mathrm{AC}$ & HR 807 & KOIII & 6.37 & 0.98 & 21 & $50 \mathrm{D} 21 \mathrm{M} 04.7 \mathrm{~S}$ & .28 \\
\hline 38 & RX J2040.6+4859 & $\mathrm{AC}$ & SAO 49928 & F9V & 9.70 & 0.60 & $20 \mathrm{H} 40 \mathrm{M} 38.29 \mathrm{~S}$ & 48D59M59.6S & .16 \\
\hline 39 & RX J2102.9+4854 & $\mathrm{AC}$ & $\mathrm{A}=\mathrm{GSC}-0359600374$ & G3V & 10.69 & - & $21 \mathrm{H} 03 \mathrm{M} 00.75 \mathrm{~S}$ & $48 \mathrm{D} 54 \mathrm{M} 28.1 \mathrm{~S}$ & .77 \\
\hline 40 & RX J2113.3+5140 & $\mathrm{AC}$ & A & $\mathrm{M} 4 \mathrm{Ve}$ & 16.50 & - & $21 \mathrm{H} 13 \mathrm{M} 23.09 \mathrm{~S}$ & $51 \mathrm{D} 40 \mathrm{M} 02.4 \mathrm{~S}$ & .34 \\
\hline 41 & RX J2123.5+4621 & $\mathrm{AC}$ & $\mathrm{A}=\mathrm{C}$ & $\mathrm{K} 4 \mathrm{~V}$ & 11.55 & - & $21 \mathrm{H} 23 \mathrm{M} 34.77 \mathrm{~S}$ & $46 \mathrm{D} 21 \mathrm{M} 40.3 \mathrm{~S}$ & .35 \\
\hline 42 & $.9+4857$ & $\mathrm{AC}$ & $\mathrm{A}=\mathrm{GSC}-0359601$ & $\mathrm{~K} 2$ & 10.51 & - & $00 \mathrm{M} 5$ & 48 & 1.04 \\
\hline 43 & RX J2130.1+4901 & $\mathrm{AC}$ & $\mathrm{B}$ & M5Ve & 14.11 & 1.59 & $4 \mathrm{~S}$ & $7 \mathrm{~S}$ & .49 \\
\hline 44 & RX J2054.1+4942 & $\mathrm{AC}$ & $\mathrm{A}=\mathrm{GSC}-0358300309$ & F8V & 10.98 & - & $20 \mathrm{H} 54 \mathrm{M} 09.16 \mathrm{~S}$ & $49 \mathrm{D} 42 \mathrm{M} 41.4 \mathrm{~S}$ & .20 \\
\hline 45 & RX J2116.6+4645 & $\mathrm{AC}$ & A & M5Ve & 15.50 & - & $21 \mathrm{H} 16 \mathrm{M} 37.23 \mathrm{~S}$ & $46 \mathrm{D} 45 \mathrm{M} 16.2 \mathrm{~S}$ & .62 \\
\hline 46 & RX J2110.2+5333 & $\mathrm{OB}$ & $\mathrm{HP}$ & Ap & 5.61 & -0.12 & $21 \mathrm{H} 10 \mathrm{M} 15.38 \mathrm{~S}$ & 53D33M47.5S & .63 \\
\hline 47 & $3+4642$ & $\mathrm{AC}$ & HR 8 & F0V & 5.60 & 0.35 & $21 \mathrm{H}$ & $46 \mathrm{D}$ & .80 \\
\hline 48 & RX J2118.5+5247 & $\mathrm{AC}$ & $\mathrm{A}=\mathrm{GSC}-0395301190$ & & 11.17 & - & $21 \mathrm{H} 18 \mathrm{M} 31.01 \mathrm{~S}$ & 52D $47 \mathrm{M} 40.8 \mathrm{~S}$ & .17 \\
\hline 49 & RX J2128.7+4409 & $? ?$ & & & - & - & & & - \\
\hline 50 & RX J2121.7+5049 & $\mathrm{AC}$ & HD 235498 & $\mathrm{~A} 0 \mathrm{~V}$ & 10.30 & 0.20 & $21 \mathrm{H} 21 \mathrm{M} 47.33 \mathrm{~S}$ & 50D49M31.6S & .47 \\
\hline 51 & RX J2132.5+4849 & $? ?$ & & & & - & & & \\
\hline 52 & $4+5023$ & $\mathrm{AC}$ & $\mathrm{A}=\mathrm{GSC}-0359700228$ & G9V & 11.55 & - & $21 \mathrm{H} 22 \mathrm{M} 26.82 \mathrm{~S}$ & $50 \mathrm{D} 23 \mathrm{M} 56.2 \mathrm{~S}$ & .92 \\
\hline 53 & RX J2035.9+4900 & $\mathrm{AC}$ & $\mathrm{A}=\mathrm{GSC}-0358101856$ & G5-8V & 10.97 & - & $20 \mathrm{H} 35 \mathrm{M} 57.27 \mathrm{~S}$ & 49D00M $43.7 \mathrm{~S}$ & .21 \\
\hline 54 & RX J2108.2+5313 & $\mathrm{AC}$ & HD 201543 & A0V & 7.30 & 0.10 & $21 \mathrm{H} 08 \mathrm{M} 17.85 \mathrm{~S}$ & 53D13M31.1S & .12 \\
\hline 55 & RX J2128.6+4653 & $\mathrm{AC}$ & $\mathrm{A}=\mathrm{GSC}-0359400075$ & K5V & 12.22 & - & $21 \mathrm{H} 28 \mathrm{M} 40.68 \mathrm{~S}$ & 46D53M56.7S & .13 \\
\hline 56 & RX J2048.0+4903 & $\mathrm{AC}$ & $\mathrm{A}=\mathrm{GSC}-0358200586$ & & 11.51 & - & $20 \mathrm{H} 48 \mathrm{M} 04.31 \mathrm{~S}$ & 49D03M12.5S & .28 \\
\hline 57 & RX J2115.4+4437 & $\mathrm{AC}$ & $\mathrm{A}=\mathrm{GSC}-0318$ & $\mathrm{~K} 0 \mathrm{~V}$ & 10.43 & - & $5 \mathrm{M} 24.00 \mathrm{~S}$ & $44 \mathrm{D} 37 \mathrm{M}$ & .62 \\
\hline 58 & RX J2109.9+4809 & $\mathrm{AC}$ & SAO 50523 & A $5 \mathrm{~V}$ & 9.10 & 0.35 & $21 \mathrm{H} 09 \mathrm{M} 54.72 \mathrm{~S}$ & 48D09M17.2S & .45 \\
\hline 59 & RX J2108.6+4927 & $\mathrm{AC}$ & A & $\mathrm{M} 2 \mathrm{Ve}$ & 14.10 & - & $21 \mathrm{H} 08 \mathrm{M} 38.91 \mathrm{~S}$ & 49D27M32.4S & .43 \\
\hline 60 & RX J2050.8+4743 & $? ?$ & & & & - & & & \\
\hline 61 & RX J2135.6+4523 & $\mathrm{AC}$ & $\mathrm{A}=\mathrm{GSC}-0359102803$ & F9V & 11.65 & - & $21 \mathrm{H} 35 \mathrm{M} 38.51 \mathrm{~S}$ & 45D23M10.6S & 1.41 \\
\hline
\end{tabular}




\begin{tabular}{|c|c|c|c|c|c|c|c|c|c|}
\hline $\begin{array}{l}\text { Source } \\
\text { index }\end{array}$ & $\begin{array}{c}\text { ROSAT } \\
\text { name }\end{array}$ & Class & Identification & $\begin{array}{l}\text { Spectral } \\
\text { type }\end{array}$ & $V$ & $B-V$ & $\begin{array}{c}\text { RA } \\
\text { (optical) }\end{array}$ & $\begin{array}{c}\text { DEC } \\
\text { (optical) }\end{array}$ & $\begin{array}{r}D_{\mathrm{X}-\mathrm{Opt}} \\
\left(\mathrm{r}_{90}\right)\end{array}$ \\
\hline 62 & RX J2119.9+5227 & $\mathrm{AC} ?$ & $? \mathrm{~A}=\mathrm{GSC}-0360101367$ & & 12.20 & - & 21H19M54.50S & 52D27M46.7S & .43 \\
\hline 63 & RX J2100.3+5219 & $\mathrm{AC}$ & $\mathrm{A}=\mathrm{GSC}-0360000514$ & M1Ve & 13.39 & - & 21H00M20.71S & 52D19M10.6S & .45 \\
\hline 64 & RX J2056.3+4817 & $? ?$ & & & - & - & & & - \\
\hline 65 & $\mathrm{RX} \mathrm{J} 2127.3+5121$ & $\mathrm{AC}$ & $\mathrm{A}+\mathrm{B}$ & & 11.46 & - & $21 \mathrm{H} 27 \mathrm{M} 20.74 \mathrm{~S}$ & 51D22M03.1S & .48 \\
\hline 66 & $\mathrm{RX} \mathrm{J} 2041.9+4746$ & $? ?$ & & & - & - & & & - \\
\hline 67 & RX J2126.3+4654 & $\mathrm{AC}$ & $\mathrm{A}=\mathrm{GSC}-0359401755$ & & 10.62 & - & $21 \mathrm{H} 26 \mathrm{M} 23.54 \mathrm{~S}$ & 46D54M31.5S & .03 \\
\hline 68 & RX J2055.8+5044 & $\mathrm{AC}$ & $\mathrm{A}=\mathrm{GSC}-0358700365$ & G8V & 12.99 & - & $20 \mathrm{H} 55 \mathrm{M} 53.12 \mathrm{~S}$ & 50D44M23.1S & .56 \\
\hline 69 & $\mathrm{RX} \mathrm{J} 2117.5+5127$ & $\mathrm{AC}$ & $\mathrm{A}=\mathrm{GSC}-0360100450$ & MoVe & 12.90 & - & $21 \mathrm{H} 17 \mathrm{M} 32.20 \mathrm{~S}$ & 51D27M14.1S & .42 \\
\hline 70 & RX J2057.7+4752 & $? ?$ & & & - & - & & & - \\
\hline 71 & RX J2131.2+4533 & $? ?$ & & & - & - & & & - \\
\hline 72 & RX J2054.6+5120 & $? ?$ & & & - & - & & & - \\
\hline 73 & $\mathrm{RX} \mathrm{J} 2121.5+4317$ & $? ?$ & & & - & - & & & - \\
\hline 74 & RX J2128.4+4900 & $? ?$ & & & - & - & & & - \\
\hline 75 & RX J2058.5+4836 & $? ?$ & & & - & - & & & - \\
\hline 76 & RX J2130.0+4740 & $\mathrm{AC} ?$ & $? \mathrm{~A}=$ Plat 2137 & $\mathrm{~K} 4 \mathrm{~V}$ & 11.98 & 1.09 & 21H30M03.89S & 47D40M25.4S & .84 \\
\hline 77 & RX J2106.2+4437 & $\mathrm{AC}$ & A & M5Ve & 15.40 & - & 21H06M16.63S & 44D37M03.8S & .39 \\
\hline 78 & RX J2128.5+4626 & $? ?$ & & & - & - & & & - \\
\hline 79 & RX J2117.7+5139 & $? ?$ & & & - & - & & & - \\
\hline 80 & RX J2101.6+4730 & $\mathrm{AC}$ & HD 200406 & G0V & 7.93 & 0.54 & 21H01M38.16S & 47D30M04.6S & 1.03 \\
\hline 81 & RX J2046.7+4728 & $\mathrm{AC} ?$ & $? \mathrm{~A}=\mathrm{GSC}-0357800892$ & & 11.20 & - & 20H46M43.30S & 47D28M33.3S & .56 \\
\hline 82 & RX J2136.6+4911 & $? ?$ & & & - & - & & & - \\
\hline 83 & RX J2118.6+5010 & $\mathrm{AC}$ & HD 203136 & K0V & 7.76 & 0.88 & $21 \mathrm{H} 18 \mathrm{M} 40.34 \mathrm{~S}$ & 50D10M57.0S & .37 \\
\hline 84 & $\mathrm{RX} \mathrm{J} 2130.3+4709$ & $\mathrm{CV}$ & $\mathrm{B}$ & $\mathrm{Me}+\mathrm{DA}$ & - & - & 21H30M18.49S & 47D10M07.6S & 1.04 \\
\hline 85 & $\mathrm{RX} \mathrm{J} 2116.0+4827$ & $? ?$ & & & - & - & & & - \\
\hline 86 & RX J2124.7+4714 & $\mathrm{AC}$ & $\mathrm{A}=\mathrm{GSC}-0359402027$ & & 10.63 & - & $21 \mathrm{H} 24 \mathrm{M} 42.66 \mathrm{~S}$ & 47D14M42.5S & .26 \\
\hline 87 & RX J2117.5+4330 & $\mathrm{AC}$ & $\mathrm{A}=\mathrm{GSC}-0318100914$ & F8V & 10.29 & - & $21 \mathrm{H} 17 \mathrm{M} 35.40 \mathrm{~S}$ & 43D30M52.1S & .95 \\
\hline 88 & RX J2137.6+4916 & $? ?$ & & & - & - & & & - \\
\hline 89 & RX J2100.6+5039 & $\mathrm{AC}$ & $\mathrm{A}=\mathrm{GSC}-0360000197$ & & 10.69 & - & 21H00M37.82S & 50D39M29.4S & .14 \\
\hline 90 & $\mathrm{RX} \mathrm{J} 2110.5+4913$ & $\mathrm{AC}$ & $\mathrm{A}=\mathrm{GSC}-0359601415$ & & 10.49 & - & 21H10M32.49S & 49D13M25.9S & .26 \\
\hline 91 & RX J2121.5+4732 & $\mathrm{AC}$ & $\mathrm{A}=\mathrm{GSC}-0359303030$ & MoVe & 12.84 & - & $21 \mathrm{H} 21 \mathrm{M} 32.55 \mathrm{~S}$ & 47D32M49.8S & .52 \\
\hline 92 & RX J2052.8+4723 & $? ?$ & & & - & - & & & - \\
\hline 93 & RX J2118.6+5039 & $? ?$ & & & - & - & & & - \\
\hline 94 & $\mathrm{RX} \mathrm{J} 2122.3+4730$ & $\mathrm{AC}$ & $\mathrm{A}=\mathrm{GSC}-0359305051$ & $\mathrm{M} 4.5 \mathrm{Ve}$ & 13.50 & - & $21 \mathrm{H} 22 \mathrm{M} 20.75 \mathrm{~S}$ & 47D30M44.6S & .24 \\
\hline 95 & RX J2050.4+4913 & $\mathrm{AC}$ & $\mathrm{A}=\mathrm{GSC}-0358300408$ & & 10.76 & - & 20H50M26.17S & 49D13M25.4S & .60 \\
\hline 96 & RX J2113.0+4834 & $\mathrm{AC}$ & $\mathrm{A}=\mathrm{GSC}-0359303505$ & G1V & 11.09 & - & $21 \mathrm{H} 13 \mathrm{M} 04.46 \mathrm{~S}$ & 48D34M23.4S & .33 \\
\hline 97 & $\mathrm{RX} \mathrm{J} 2134.2+4911$ & $\mathrm{AC} ?$ & $? \mathrm{~A}=$ Plat 5991 & M2-M6 & 12.62 & 1.49 & 21H34M16.99S & 49D11M21.4S & .56 \\
\hline 98 & RX J2119.5+4351 & $? ?$ & & & - & - & & & - \\
\hline 99 & RX J2117.4+5152 & $? ?$ & & & - & - & & & - \\
\hline 100 & $\mathrm{RX} \mathrm{J} 2123.2+5057$ & $? ?$ & & & - & - & & & - \\
\hline 101 & RX J2053.4+4759 & $? ?$ & & & - & - & & & - \\
\hline 102 & RX J2124.3+5059 & $\mathrm{AC}$ & $\mathrm{A}$ & $\mathrm{K} 7 \mathrm{Ve}$ & 13.30 & - & $21 \mathrm{H} 24 \mathrm{M} 17.95 \mathrm{~S}$ & 50D59M46.4S & .28 \\
\hline 103 & RX J2114.1+4840 & $\mathrm{AC} ?$ & $? \mathrm{~A}+\mathrm{B}$ & & 11.36 & - & 21H14M09.20S & 48D40M53.0S & .90 \\
\hline 104 & RX J2043.7+4727 & $\mathrm{AC} ?$ & $? \mathrm{~A}=\mathrm{GSC}-0357801789$ & & 11.70 & - & 20H43M47.00S & 47D27M19.4S & .90 \\
\hline 105 & RX J2122.6+4956 & $? ?$ & & & - & - & & & - \\
\hline 106 & RX J2103.6+4845 & $\mathrm{AC}$ & $\mathrm{A}=\mathrm{GSC}-0359600092$ & & 10.65 & - & 21H03M37.95S & 48D45M40.6S & .73 \\
\hline 107 & RX J2059.8+4937 & $\mathrm{AC} ?$ & $? \mathrm{~A}=\mathrm{GSC}-0358300546$ & & 12.58 & - & $20 \mathrm{H} 59 \mathrm{M} 50.22 \mathrm{~S}$ & 49D37M30.9S & .45 \\
\hline 108 & RX J2126.9+4636 & $? ?$ & & & - & - & & & - \\
\hline 109 & RX J2120.2+5127 & $\mathrm{AC}$ & $\mathrm{A}=\mathrm{GSC}-0360100440$ & & 11.08 & - & $21 \mathrm{H} 20 \mathrm{M} 17.38 \mathrm{~S}$ & 51D27M44.2S & .11 \\
\hline 110 & RX J2130.7+4919 & $\mathrm{AC}$ & $\mathrm{A}=$ Plat 2930 & F9 & 12.44 & 0.56 & 21H30M43.51S & 49D18M59.7S & .88 \\
\hline 111 & RX J2123.7+4636 & $? ?$ & & & - & - & & & - \\
\hline 112 & RX J2043.4+5002 & $\mathrm{AC} ?$ & $? \mathrm{~A}=\mathrm{GSC}-0358200283$ & & 12.90 & - & $20 \mathrm{H} 43 \mathrm{M} 29.31 \mathrm{~S}$ & 50D02M13.3S & .88 \\
\hline 113 & RX J2107.6+5048 & $\mathrm{AC}$ & $\mathrm{A}=\mathrm{GSC}-0360000137$ & G1V & 11.31 & - & $21 \mathrm{H} 07 \mathrm{M} 37.64 \mathrm{~S}$ & 50D48M60.0S & .51 \\
\hline 114 & RX J2117.5+5243 & $\mathrm{AC} ?$ & $? \mathrm{~A}=\mathrm{GSC}-0395301075$ & & 12.62 & - & $21 \mathrm{H} 17 \mathrm{M} 31.78 \mathrm{~S}$ & 52D43M07.5S & .17 \\
\hline 115 & RX J2120.8+5209 & $? ?$ & & & - & - & & & - \\
\hline 116 & RX J2121.8+5135 & $\mathrm{AC}$ & $\mathrm{A}=\mathrm{GSC}-0360100693$ & & 11.47 & - & $21 \mathrm{H} 21 \mathrm{M} 52.28 \mathrm{~S}$ & 51D35M18.0S & .29 \\
\hline 117 & RX J2121.0+5049 & $? ?$ & & & - & - & & & - \\
\hline 118 & RX J2058.1+4552 & $\mathrm{AC} ?$ & $? \mathrm{~A}=\mathrm{GSC}-0357501843$ & & 10.67 & - & $20 \mathrm{H} 58 \mathrm{M} 11.67 \mathrm{~S}$ & 45D52M49.1S & .35 \\
\hline 119 & RX J2112.9+5314 & $\mathrm{AC}$ & $\mathrm{A}=\mathrm{GSC}-0395300355$ & & 9.95 & - & $21 \mathrm{H} 12 \mathrm{M} 56.17 \mathrm{~S}$ & 53D14M31.1S & .24 \\
\hline 120 & RX J2122.6+4856 & $\mathrm{AC} ?$ & $? \mathrm{~A}=\mathrm{GSC}-0359800927$ & & 11.37 & - & $21 \mathrm{H} 22 \mathrm{M} 37.38 \mathrm{~S}$ & 48D56M19.0S & .19 \\
\hline 121 & RX J2122.0+5059 & $? ?$ & & & - & - & & & - \\
\hline 122 & RX J2105.7+4933 & $? ?$ & & & - & - & & & - \\
\hline 123 & RX J2116.0+5254 & $? ?$ & & & - & - & & & - \\
\hline 124 & RX J2059.6+4731 & $\mathrm{AC} ?$ & $? \mathrm{~A}=\mathrm{GSC}-0357901518$ & & 12.47 & - & $20 \mathrm{H} 59 \mathrm{M} 40.43 \mathrm{~S}$ & 47D31M09.6S & .14 \\
\hline 125 & RX J2100.8+5213 & $\mathrm{AC} ?$ & $? \mathrm{~A}=\mathrm{GSC}-0360000242$ & & 13.27 & - & 21H00M52.05S & 52D13M58.8S & .03 \\
\hline 126 & RX J2111.8+4942 & $? ?$ & & & - & - & & & - \\
\hline 127 & RX J2108.9+4958 & $? ?$ & & & - & - & & & - \\
\hline 128 & RX J2122.9+4941 & $? ?$ & & & - & - & & & - \\
\hline
\end{tabular}


Table 5. Optical properties of all proposed optical counterparts in the Cygnus test region for sources with $7 \leq M L<8$. The last column lists the distance between X-ray an optical positions in units of the $90 \%$ confidence radius. For the sake of completeness, we also list the proposed counterparts having a probability of identification in the range $95 \%-98 \%$. These more uncertain cases are marked by a "?" as first letter of the identification name and the class acronym is followed by a "?"

\begin{tabular}{|c|c|c|c|c|c|c|c|c|c|}
\hline $\begin{array}{l}\text { Source } \\
\text { index }\end{array}$ & $\begin{array}{c}\text { ROSAT } \\
\text { name }\end{array}$ & Class & Identification & $\begin{array}{l}\text { Spectral } \\
\text { type }\end{array}$ & V & $B-V$ & $\begin{array}{c}\text { RA } \\
\text { (optical) }\end{array}$ & $\begin{array}{c}\text { DEC } \\
\text { (optical) }\end{array}$ & $\begin{array}{r}D \mathrm{X}-\mathrm{Opt} \\
\left(\mathrm{r}_{90}\right)\end{array}$ \\
\hline 129 & RX J2053.2+4951 & ?? & & & - & - & & & - \\
\hline 130 & RX J2054.0+4858 & $? ?$ & & & - & - & & & - \\
\hline 131 & RX J2049.6+5128 & $\mathrm{AC}$ & $\mathrm{A}=\mathrm{GSC}-0358700147$ & & 11.53 & - & $20 \mathrm{H} 49 \mathrm{M} 39.75 \mathrm{~S}$ & $51 \mathrm{D} 28 \mathrm{M} 34.1 \mathrm{~S}$ & 26 \\
\hline 132 & RX J2052.3+4843 & $\mathrm{AC}$ & $\mathrm{A}=\mathrm{GSC}-0357901214$ & & 9.75 & - & $20 \mathrm{H} 52 \mathrm{M} 20.16 \mathrm{~S}$ & 48D $43 \mathrm{M} 26.5 \mathrm{~S}$ & .36 \\
\hline 133 & RX J2129.2+4604 & ?? & & & - & - & & & - \\
\hline 134 & RX J2124.5+5133 & $? ?$ & & & - & - & & & - \\
\hline 135 & $\mathrm{RX} \mathrm{J} 2117.2+5241$ & $\mathrm{AC}$ & $\mathrm{A}=\mathrm{GSC}-0395301223$ & & 10.42 & - & $21 \mathrm{H} 17 \mathrm{M} 11.64 \mathrm{~S}$ & $52 \mathrm{D} 40 \mathrm{M} 41.8 \mathrm{~S}$ & 1.01 \\
\hline 136 & RX J2057.6+4750 & $? ?$ & & & - & - & & & - \\
\hline 137 & RX J2111.6+4809 & $\mathrm{CV}$ & V1500 Cyg & & 17.06 & 0.38 & $21 \mathrm{H} 11 \mathrm{M} 36.60 \mathrm{~S}$ & 48D09M02.0S & .51 \\
\hline 138 & RX J2051.8+4741 & $? ?$ & & & - & - & & & - \\
\hline 139 & RX J2057.1+4929 & $? ?$ & & & - & - & & & - \\
\hline 140 & RX J2043.1+4752 & $\mathrm{AC} ?$ & $? \mathrm{~A}=\mathrm{GSC}-0357802133$ & & 11.42 & - & $20 \mathrm{H} 43 \mathrm{M} 08.36 \mathrm{~S}$ & $47 \mathrm{D} 52 \mathrm{M} 13.8 \mathrm{~S}$ & .48 \\
\hline 141 & RX J2127.1+4801 & $? ?$ & & & - & - & & & - \\
\hline 142 & RX J2048.8+4631 & ?? & & & - & - & & & - \\
\hline 143 & RX J2110.5+5244 & $? ?$ & & & - & - & & & - \\
\hline 144 & RX J2052.3+4656 & ?? & & & - & - & & & - \\
\hline 145 & RX J2101.2+4609 & OB & HD 200310 & $\mathrm{~B} 1 \mathrm{Ve}$ & 5.40 & -0.18 & $21 \mathrm{H} 01 \mathrm{M} 10.80 \mathrm{~S}$ & 46D09M20.8S & .61 \\
\hline 146 & RX J2055.7+4815 & ?? & & & - & - & & & - \\
\hline 147 & RX J2045.1+4805 & $\mathrm{AC}$ & $\mathrm{A}=\mathrm{GSC}-0357800263$ & & 11.37 & - & $20 \mathrm{H} 45 \mathrm{M} 10.39 \mathrm{~S}$ & 48D05M12.3S & .25 \\
\hline 148 & RX J2110.3+4832 & ?? & & & - & - & & & - \\
\hline 149 & RX J2131.2+4812 & ?? & & & - & - & & & - \\
\hline 150 & RX J2058.7+4845 & $? ?$ & & & - & - & & & - \\
\hline 151 & RX J2100.3+4810 & ?? & & & - & - & & & - \\
\hline 152 & RX J2130.8+4842 & ?? & & & - & - & & & - \\
\hline 153 & RX J2052.6+4703 & ?? & & & - & - & & & - \\
\hline 154 & RX J2055.0+5039 & $? ?$ & & & - & - & & & - \\
\hline 155 & RX J2121.1+4907 & $? ?$ & & & - & - & & & - \\
\hline 156 & RX J2122.4+4709 & $? ?$ & & & - & - & & & - \\
\hline 157 & RX J2054.6+4903 & ?? & & & - & - & & & - \\
\hline 158 & RX J2121.5+4951 & ?? & & & - & - & & & - \\
\hline
\end{tabular}


Table 6. Notes on individual sources

\begin{tabular}{|c|c|c|}
\hline $\begin{array}{r}\text { Source } \\
\text { index } \\
\end{array}$ & $\begin{array}{c}\text { ROSAT } \\
\text { name }\end{array}$ & Note \\
\hline 1 & RX J2112.7+5006 & GD $394=$ RE J211241+500619 \\
\hline 2 & RX J2133.9+4535 & $\mathrm{HD} 205435=\mathrm{HR} 8252=\rho \mathrm{Cyg}$ \\
\hline 6 & RX J2106.0+5421 & In ADS catalogue \\
\hline 8 & RX J2130.8+4827 & Member of NGC 7092, in ADS catalogue \\
\hline 10 & RX J2124.7+4639 & GSC-0359001727 merges objects A and B \\
\hline 11 & RX J2117.3+5044 & $\begin{array}{l}\text { Our candidate was independently discovered by Lanning, H.H. and Meakes, M. (1994; } \\
\text { PASP, 106, 38) in a search for faint UV-bright stars in the galactic plane. A finding } \\
\text { chart may be found in their work. }\end{array}$ \\
\hline 12 & RX J2135.9+4728 & $z=0.025$ \\
\hline 13 & RX J2102.6+4552 & $\begin{array}{l}\text { Chromospheric activity in HD } 200560 \text { was reported by Soderblom (1985, AJ, 90, } \\
\text { 2103). }\end{array}$ \\
\hline 15 & RX J2055.3+5025 & $\begin{array}{l}\text { On the basis of its position outside the } 90 \% \text { confidence circle and weak Ca II H\&K } \\
\text { emission, star B is unlikely to contribute much to the X-ray source. The finding chart } \\
\text { was extracted from the STSCI digitized sky survey. }\end{array}$ \\
\hline 23 & RX J2100.1+4841 & $\mathrm{BD}+483260 \mathrm{E}=\mathrm{ADS} 14531 \mathrm{E}$ \\
\hline 26 & RX J2107.3+5202 & $\begin{array}{l}\text { This star is an eclipsing binary of the Algol type with an orbital period of } 2.3467 \\
\text { days. }\end{array}$ \\
\hline 30 & RX J2120.4+4733 & $=\mathrm{ADS} 14886 \mathrm{AB}$ \\
\hline 37 & RX J2103.4+5021 & $\begin{array}{l}\text { Ca H\&K emission also visible in the high resolution spectrum of Strassmeier (1994, } \\
\text { A\&AS, 103, 413). }\end{array}$ \\
\hline 38 & RX J2040.6+4859 & $=\mathrm{ADS} 14161 \mathrm{~A}$ \\
\hline 43 & RX J2130.1+4901 & $\begin{array}{l}\text { Candidate object 'B' coincides in position with star } 2376 \text { in the catalogue of stars in } \\
\text { the region of the open cluster M39 compiled by Platais (1994). This M5Ve star lies } \\
\text { far off the photometric sequence defined by Mohan and Sagar (1985, MNRAS, 213, } \\
337 \text { ) for this cluster and we conclude that object "B" is a foreground star not related } \\
\text { to the open cluster. Object "A" would deserve follow-up observations. }\end{array}$ \\
\hline 51 & RX J2132.5+4849 & $\begin{array}{l}\text { Candidates A and B are respectively stars } 4522 \text { and } 4516 \text { in the catalogue of stars } \\
\text { in the region of the open cluster M } 39 \text { compiled by Platais (1994). Our high and } \\
\text { low resolution (not shown) spectra show that A is a G1 star and B is a K7 star. } \\
\text { These spectral types are in agreement with the } B-V \text { indices listed in Platais (1994). } \\
\text { Assuming that the two stars are on the main sequence implies a distance modulus } \\
\text { incompatible with a membership to M39. Recent additional optical observations (not } \\
\text { shown) indicate that the two brightest objects east of "B" (D and E not marked) are } \\
\text { M stars with weak H } \alpha \text { emission. The X-ray source could result from the combined } \\
\text { coronal emission of several of the stars held in the error circle. }\end{array}$ \\
\hline 53 & RX J2035.9+4900 & $\begin{array}{l}\text { The medium resolution spectrum of object "A", the proposed optical counterpart, } \\
\text { exhibits clear line doubling revealing the presence of a close binary. }\end{array}$ \\
\hline 58 & RX J2109.9+4809 & $\begin{array}{l}\text { The } U B V \text { photometry reported by Young et al. }(1976, \text { ApJ, 209, 882) suggests a } \\
\text { spectral type slightly later than A5 for SAO } 50523 .\end{array}$ \\
\hline 65 & RX J2127.3+5121 & $\begin{array}{l}\text { Object A is GSC-0360200451 }(V=11.92) \text { and B is GSC-0360200459 }(V=11.46) \text {. } \\
\text { Optical coordinates and magnitudes recorded in Tables are those of } B .\end{array}$ \\
\hline
\end{tabular}




\begin{tabular}{|c|c|c|}
\hline $\begin{array}{r}\text { Source } \\
\text { index }\end{array}$ & $\begin{array}{l}\text { ROSAT } \\
\text { name }\end{array}$ & Note \\
\hline 68 & RX J2055.8+5044 & X-ray position compatible with IRAS $20544+5033$ \\
\hline 74 & RX J2128.4+4900 & $\begin{array}{l}\text { Star A }(V=13.75 ; B-V=0.57 ; U-B=0.45) \text { is number } 889 \text { in the catalog of } \\
\text { objects in the direction of M39 by Platais }(1994) \text {. }\end{array}$ \\
\hline 76 & RX J2130.0+4740 & $\begin{array}{l}\text { Candidates A }(V=11.98 ; B-V=1.09 ; U-B=0.68) \text { and } \mathrm{B}(V=11.00 ; B-V= \\
0.52 ; U-B=-0.10 \text {, spectral type } \mathrm{F} 6) \text { are respectively stars } 2137 \text { and } 2189 \text { in the } \\
\text { catalog of objects in the region of M39 (Platais 1994). The colour indices of object } \\
\text { A suggest a K4 spectral type with a distance modulus smaller than M39 whereas } \\
\text { candidate B is likely to belong to the open cluster. }\end{array}$ \\
\hline 92 & RX J2052.8+4723 & X-ray position compatible with IRAS $20512+4712$. \\
\hline 97 & RX J2134.2+4911 & $\begin{array}{l}\text { Candidate A is number } 5991 \text { in the catalog of objects in the region of M39 (Platais } \\
\text { 1994). Its red colours are compatible with A being a nearby M2-M6 star. Two other } \\
\text { stars in Platais's list ( } 5981 \text { and } 5999 \text { ) fall inside the } 90 \% \text { confidence radius. } U B V \\
\text { photometry suggests that these two objects are G type stars located behind M39 and } \\
\text { are therefore less likely optical counterparts of the X-ray source. }\end{array}$ \\
\hline 103 & RX J2114.1+4840 & $\begin{array}{l}\text { Object A is GSC-0359304712 }(V=11.36) \text { and Object B is GSC-0359304650 }(V= \\
13.61) . \text { The optical positions and magnitudes recorded in Tables are those of A. }\end{array}$ \\
\hline 105 & RX J2122.6+4956 & X-ray position compatible with IRAS $21209+4943$. \\
\hline 108 & RX J2126.9+4636 & X-ray position compatible with IRAS $21252+4624$ \\
\hline 141 & RX J2127.1+4801 & $\begin{array}{l}\text { A possible identification based on positional coincidence is star } 173 \text { in the catalogue } \\
\text { of objects in the region of M39 (Platais 1994). }\end{array}$ \\
\hline 149 & RX J2131.2+4812 & $\begin{array}{l}\text { The GSC entry closest to the X-ray position is star } 3359 \text { in the catalogue of objects } \\
\text { in the region of M39 (Platais 1994). Its colour suggests a G2-G5 type and a distance } \\
\text { compatible with a membership to M39. }\end{array}$ \\
\hline 152 & RX J2130.8+4842 & $\begin{array}{l}\text { Star number } 3016 \text { of the catalog of objects in the region of M39 (Platais 1994) falls } \\
\text { into the } 90 \% \text { confidence error circle. The } U B V \text { photometry is consistent with a K2-K5 } \\
\text { dwarf in M39. }\end{array}$ \\
\hline
\end{tabular}

